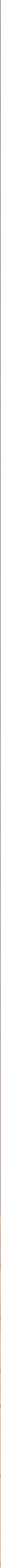




\section{Néron's pairing and relative algebraic equivalence}

\section{Cédric Pépin}

Let $R$ be a complete discrete valuation ring with algebraically closed residue field $k$ and fraction field $K$. Let $X_{K}$ be a proper smooth and geometrically connected scheme over $K$. Néron defined a canonical pairing on $X_{K}$ between 0-cycles of degree zero and divisors which are algebraically equivalent to zero. When $X_{K}$ is an abelian variety, and if one restricts to those 0-cycles supported on $K$ rational points, Néron gave an expression of his pairing involving intersection multiplicities on the Néron model $A$ of $A_{K}$ over $R$. When $X_{K}$ is a curve, Gross and Hriljac gave independently an analogous description of Néron's pairing, but for arbitrary 0 -cycles of degree zero, by means of intersection theory on a proper flat regular $R$-model $X$ of $X_{K}$.

We show that these intersection computations are valid for an arbitrary scheme $X_{K}$ as above and arbitrary 0-cycles of degree zero, by using a proper flat normal and semifactorial model $X$ of $X_{K}$ over $R$. When $X_{K}=A_{K}$ is an abelian variety, and $X=\bar{A}$ is a semifactorial compactification of its Néron model $A$, these computations can be used to study the relative algebraic equivalence on $\bar{A} / R$. We then obtain an interpretation of Grothendieck's duality for the Néron model $A$, in terms of the Picard functor of $\bar{A}$ over $R$. Finally, we give an explicit description of Grothendieck's duality pairing when $A_{K}$ is the Jacobian of a curve of index one.

1. Introduction

2. Néron's pairing and intersection multiplicities

3. Duality and algebraic equivalence for models of abelian varieties 1329

4. Grothendieck's pairing for Jacobians

Acknowledgments

References

\section{Introduction}

Let $R$ be a complete discrete valuation ring with algebraically closed residue field $k$ and fraction field $K$. Let $X_{K}$ be a proper smooth and geometrically connected

Partially supported by the FWO project ZKC1235-PS-011 G.0415.10 at KU Leuven. MSC2010: primary 14K30; secondary 14G40, 14K15, $11 \mathrm{G} 10$.

Keywords: Néron's symbol, Picard functor, Néron models, duality, Grothendieck's pairing. 
scheme over $K$. Denote by $Z_{0}^{0}\left(X_{K}\right)$ the group of 0 -cycles of degree zero on $X_{K}$, and by $\operatorname{Div}^{0}\left(X_{K}\right)$ the group of divisors which are algebraically equivalent to zero on $X_{K}$. For each $c_{K} \in Z_{0}^{0}\left(X_{K}\right)$ and $D_{K} \in \operatorname{Div}^{0}\left(X_{K}\right)$ with disjoint supports, Néron attached a rational number

$$
\left\langle c_{K}, D_{K}\right\rangle \in \mathbb{Q},
$$

by using the unique (up to constant) Néron function associated to $D_{K}$. This defines a bilinear pairing $\langle\cdot, \cdot\rangle$; see [Néron 1965, II 9.3].

Suppose first that $X_{K}=A_{K}$ is an abelian variety, and denote by $A$ its Néron model over $R$. By definition of $A$, any $K$-rational point of $A_{K}$ extends to a section of $A$ over $R$. Then, if $c_{K}$ is supported on $K$-rational points, Néron showed that the pairing attached to $A_{K}$ can be decomposed as follows:

$$
\left\langle c_{K}, D_{K}\right\rangle=i\left(c_{K}, D_{K}\right)+j\left(c_{K}, D_{K}\right),
$$

where $i\left(c_{K}, D_{K}\right)$ is the intersection multiplicity $\left(\bar{c}_{K} \cdot \bar{D}_{K}\right) \in \mathbb{Z}$ of the schematic closures in $A$, and $j\left(c_{K}, D_{K}\right) \in \mathbb{Q}$ depends only on the specialization of $c_{K}$ on the group $\Phi_{A}$ of connected components of the special fiber $A_{k}$; see [Néron 1965, III 4.1; Lang 1983, 11.5.1].

Suppose now that $X_{K}$ is a curve, and denote by $X$ a proper flat regular model of $X_{K}$ over $R$. Let $M$ be the intersection matrix of the special fiber $X_{k}$ of $X / R$ : if $\Gamma_{1}, \ldots, \Gamma_{v}$ are the irreducible components of $X_{k}$ equipped with their reduced scheme structure, the $(i, j)$-th entry of $M$ is the intersection number $\left(\Gamma_{i} \cdot \Gamma_{j}\right)$. Let $D_{K} \in \operatorname{Div}^{0}\left(X_{K}\right)$ and let $\bar{D}_{K}$ be its closure in $X$. Computing the degree $\left(\bar{D}_{K} \cdot \Gamma_{i}\right)$ of $\bar{D}_{K}$ along each $\Gamma_{i}$, we get a vector $\rho\left(\bar{D}_{K}\right) \in \mathbb{Z}^{v}$. Next, as a consequence of intersection theory on $X$, there exists a vector $V \in \mathbb{Q}^{v}$ such that $\rho\left(\bar{D}_{K}\right)=M V$. Denote again by $V$ the $\mathbb{Q}$-linear combination of the $\Gamma_{i}$ where the coefficient of $\Gamma_{i}$ is the $i$-th entry of $V$. Then, for any $c_{K} \in Z_{0}^{0}\left(X_{K}\right)$ whose support is disjoint from that of $D_{K}$, the following formula holds:

$$
\left\langle c_{K}, D_{K}\right\rangle=\left(\bar{c}_{K} \cdot \bar{D}_{K}\right)+\left(\bar{c}_{K} \cdot(-V)\right),
$$

where the second intersection number is defined by $\mathbb{Q}$-linearity from the $\left(\bar{c}_{K} \cdot \Gamma_{i}\right)$. See [Gross 1986; Hriljac 1985; Lang 1988, III 5.2]. Now let $J_{K}$ be the Jacobian of $X_{K}$ and let $J$ be its Néron model over $R$. Following the point of view of Bosch and Lorenzini [2002, 4.3], it results from Raynaud's theory of the Picard functor $\operatorname{Pic}_{X / R}$ [Raynaud 1970, Section 8] that the term $\left(\bar{c}_{K} \cdot(-V)\right)$ depends only on the specialization of $\left(c_{K}\right) \in J_{K}(K)$ into the group of components $\Phi_{J}$ of $J_{k}$.

In Section 2, we provide a unified approach to these two descriptions of Néron's pairing. More precisely, for an arbitrary proper geometrically normal and geometrically connected scheme $X_{K}$, there always exists some proper flat normal semifactorial model $X$ of $X_{K}$ over $R$ [Pepin 2011, Theorem 2.6]. Recall that $X / R$ is 
semifactorial if the restriction homomorphism on Picard groups $\operatorname{Pic}(X) \rightarrow \operatorname{Pic}\left(X_{K}\right)$ is surjective. Note that a regular model is semifactorial. Using the theory of the Picard functor of semifactorial models, we define a pairing $[\cdot, \cdot]$ on $X_{K}$ involving intersection multiplicities on $X$ (Definition 2.1.1). It turns out that this pairing depends only on $X_{K}$, and coincides with Néron's pairing when the latter is defined, that is, when $X_{K}$ is smooth:

$$
\langle\cdot, \cdot\rangle=[\cdot, \cdot]
$$

(Theorem 2.2.1). If $X_{K}=A_{K}$ is an abelian variety and $X=\bar{A}$ is a semifactorial compactification of its Néron model $A$, then equality (3) provides decomposition (1) for 0-cycles supported on $K$-rational points. If $X_{K}$ is a curve and $X$ a proper flat regular model of $X_{K}$, then the intersection matrix of $X_{k}$ is defined, and equality (3) is exactly formula (2).

In Section 3, we consider an abelian variety $A_{K}$, with dual $A_{K}^{\prime}$. By definition, the abelian variety $A_{K}^{\prime}$ parametrizes the divisors on $A_{K}$ which are algebraically equivalent to zero, that is, $A_{K}^{\prime}=\operatorname{Pic}_{A_{K} / K}^{0}$. Now, let $A^{\prime} / R$ be the Néron model of $A_{K}^{\prime}$, and denote by $\left(A^{\prime}\right)^{0}$ its identity component. By restricting to the generic fiber, the group of sections $\left(A^{\prime}\right)^{0}(R)$ can be viewed as a subgroup of $A_{K}^{\prime}(K)$. On the other hand, let $\bar{A}$ be a normal semifactorial compactification of $A$, let $\mathrm{Pic}_{\bar{A} / R}$ be its relative Picard functor, and let $\mathrm{Pic}_{\bar{A} / R}^{0}$ be the component of the zero section. By restricting to the generic fiber, the group $\operatorname{Pic}_{\bar{A} / R}^{0}(R)$ can be viewed as a subgroup of $\operatorname{Pic}_{A_{K} / K}^{0}(K)$.

In Theorem 3.2.1, we investigate the relationship between the two groups

$$
\left(A^{\prime}\right)^{0}(R) \quad \text { and } \quad \operatorname{Pic}_{\bar{A} / R}^{0}(R) \quad\left(\text { contained in } A_{K}^{\prime}(K)=\operatorname{Pic}_{A_{K} / K}^{0}(K)\right) .
$$

We show that they are equal as soon as the duality conjecture of Grothendieck about $A$ and $A^{\prime}$ is true [SGA 7 I 1972, IX 1.3]. More precisely, Grothendieck defined a pairing between the component groups of the special fibers of $A$ and $A^{\prime}$, and he conjectured that this pairing is perfect. This duality statement has been proved in many situations (see the introduction of [Bosch and Lorenzini 2002] for a detailed list of the known cases, and also [Loerke 2009]), but it remains open in equal characteristic $p>0$. Here, we give an equivalent formulation of Grothendieck's conjecture, in terms of Cartier divisors on $\bar{A}$. As a consequence, when the conjecture is true, we obtain the equality $\left(A^{\prime}\right)^{0}(R)=\operatorname{Pic}_{\bar{A} / R}^{0}(R)$. As a Cartier divisor on $\bar{A}$ is said to be algebraically equivalent to zero relative to $R$ if its image into $\operatorname{Pic}_{\bar{A} / R}(R)$ is contained $\operatorname{Pic}_{\bar{A} / R}^{0}(R)$, the latter equality says that these divisors are parametrized by $\left(A^{\prime}\right)^{0}$. The main ingredients for the proof are a theorem of Bosch and Lorenzini about Néron's and Grothendieck's pairings [Bosch and Lorenzini 2002, 4.4], and the study of the pairing $[\cdot, \cdot]$ introduced above, especially for 0 -cycles supported on nonrational points (Proposition 3.4.2). 
In Section 4, we examine the relationship between Néron's and Grothendieck's pairing for the Jacobian of a curve, following Bosch and Lorenzini [2002, 4.6] and Lorenzini [2008, 3.4]. Here we take into account the index of the curve (Theorem 4.1.1). As a consequence, we obtain the perfectness of Grothendieck's pairing when this index is prime to the characteristic of the residue field $k$ (Corollary 4.1.2).

\section{Néron's pairing and intersection multiplicities}

In this article, let us adopt the following terminology: a divisor on a scheme will always be a Cartier divisor.

2.1. A canonical pairing computed on semifactorial models. Let $R$ be a discrete valuation ring with fraction field $K$ and residue field $k$. We assume $R$ complete and $k$ algebraically closed. Let $X_{K}$ be a proper geometrically normal and geometrically connected scheme over $K$. From [Pepin 2011, Theorem 2.6], there exists a model $X / R$ of $X_{K}$, that is, an $R$-scheme with generic fiber $X_{K}$, which is proper, flat, normal and semifactorial: every invertible sheaf on $X_{K}$ can be extended to an invertible sheaf on $X$. To each 0 -cycle $c_{K} \in Z_{0}^{0}\left(X_{K}\right)$ and divisor $D_{K} \in \operatorname{Div}^{0}\left(X_{K}\right)$ with support disjoint from that of $c_{K}$, we will attach a number $\left[c_{K}, D_{K}\right]_{X} \in \mathbb{Q}$ using intersection multiplicities on $X$. For this purpose, let us first recall some definitions and one result.

Intersection multiplicities. Let $X / R$ be a proper $R$-scheme. Let $c_{K}$ be a 0 -cycle on the generic fiber $X_{K}$. Denote by $\bar{c}_{K}$ its schematic closure in $X$ : if $c_{K}=\sum_{i} n_{i}\left[x_{K, i}\right]$, then $\bar{c}_{K}=\sum_{i} n_{i}\left[\bar{x}_{K, i}\right]$, where $\bar{x}_{K, i}$ is the closure in $X$ of the closed point $x_{K, i}$ of $X_{K}$. On the other hand, let $\Delta$ be a divisor on $X$ whose support does not meet that of $c_{K}$. The intersection multiplicity $\left(\bar{c}_{K} \cdot \Delta\right)$ of $\bar{c}_{K}$ and $\Delta$ on $X$ is defined as follows. Let $x_{K}$ be a point of the support of $c_{K}$. Let $Z:=\bar{x}_{K}$ be its schematic closure in $X$. This is an integral scheme, finite and flat over $R$, which is local because $R$ is henselian. Set $x_{k}:=Z \cap X_{k}$. If $f \in K(X)$ is a local equation for $\Delta$ in a neighborhood of $x_{k}$, then $\left(\bar{c}_{K} \cdot \Delta\right)_{x_{k}}$ is the order of $\left.f\right|_{Z}$ at $x_{k}$ : writing $\left.f\right|_{Z}=a / b$ with regular $a, b \in \mathbb{O}(Z)$, then

$$
\left(\bar{c}_{K} \cdot \Delta\right)_{x_{k}}=\text { length }_{\mathscr{O}(Z)}(\mathcal{O}(Z) /(a))-\text { length }_{\mathscr{O}(Z)}(\mathcal{O}(Z) /(b))
$$

[Fulton 1998, page 8]. The whole intersection multiplicity $\left(\bar{c}_{K} \cdot \Delta\right)$ is defined by $\mathbb{Z}$-linearity.

Let us also give another description of $\left(\bar{c}_{K} \cdot \Delta\right)_{x_{k}}$, which will be useful in the sequel. As $R$ is excellent, the normalization $\widetilde{Z} \rightarrow Z$ is finite. Moreover, as $k$ is algebraically closed,

$$
\text { length }_{\mathscr{O}(Z)}(\mathscr{O}(Z) /(a))=\text { length }_{R}(\mathcal{O}(Z) /(a)),
$$


for any regular $a \in \mathcal{O}(Z)$, and the same formula holds with $Z$ replaced by $\widetilde{Z}$ [loc. cit., Appendix A.1.3]. But

$$
\text { length }_{R}(\mathcal{O}(Z) /(a))=\operatorname{length}_{R}(\mathcal{O}(\widetilde{Z}) /(a))
$$

for any regular $a \in \mathcal{O}(Z)$; see [Bosch et al. 1990, end of page 237]. Thus, if $f \in K(X)$ is a local equation for $\Delta$ in a neighborhood of $x_{k}$, we have obtained that

$$
\left(\bar{c}_{K} \cdot \Delta\right)_{x_{k}}=\left\{\begin{array}{cl}
\operatorname{length}_{\mathscr{O}(\widetilde{Z})}(\mathscr{O}(\widetilde{Z}) /(f)) & \text { if }\left.f\right|_{\tilde{Z}} \in \mathcal{O}(\widetilde{Z}), \\
-\operatorname{length}_{\mathscr{O}(\widetilde{Z})}\left(\mathscr{O}(\widetilde{Z}) /\left(f^{-1}\right)\right) & \text { otherwise. }
\end{array}\right.
$$

Relative algebraic equivalence and relative $\tau$-equivalence [Raynaud 1970, 3.2d; SGA 6 1971, XIII 4]. If $G$ is a commutative group scheme locally of finite type over a field, the identity component $G^{0}$ of $G$ is the open subscheme of $G$ whose underlying topological space is the connected component of the identity element of $G$. The $\tau$-component of $G$ is open subgroup scheme $G^{\tau}$ of $G$ which is the inverse image of the torsion subgroup of $G / G^{0}$. When $G$ is a commutative group functor over a scheme $T$, whose fibers are representable by schemes locally of finite type, the identity component and $\tau$-component of $G$ are the subfunctors $G^{\tau}$ of $G$ whose fibers are the $G_{t}^{0}, t \in T$ and $G_{t}^{\tau}, t \in T$, respectively. Note that $G^{0} \subseteq G^{\tau}$.

Let $Z \rightarrow T$ be a proper morphism of schemes. Then the fibers of the Picard functor $\mathrm{Pic}_{Z / T}$ are representable by schemes locally of finite type [Murre 1964; Oort 1962]. Let $\mathscr{L}$ be an invertible $\mathscr{O}_{Z}$-module. The sheaf $\mathscr{L}$ is said to be algebraically equivalent to zero relative to $T$ if its image into $\operatorname{Pic}_{Z / T}(T)$ belongs to the subgroup $\operatorname{Pic}_{Z / T}^{0}(T)$, that is $\mathscr{L}_{t} \in \mathrm{Pic}_{Z_{t} / t}^{0}(t)$ for all $t \in T$. When there is no ambiguity about the base scheme $T$, we will just say that $\mathscr{L}$ is algebraically equivalent to zero. Similarly, the sheaf $\mathscr{L}$ is said to be $\tau$-equivalent to zero relative to $T$ if its image into $\operatorname{Pic}_{Z / T}(T)$ belongs to the subgroup $\operatorname{Pic}_{Z / T}^{\tau}(T)$, that is $\mathscr{L}_{t} \in \operatorname{Pic}_{Z_{t} / t}^{\tau}(t)$ for all $t \in T$. If $D$ is a divisor on $Z$, it is algebraically equivalent to zero, or $\tau$-equivalent to zero, respectively, relative to $T$ if the associated invertible sheaf $\mathrm{O}_{Z}(D)$ is. We denote by $\operatorname{Div}^{0}(Z / T)$ and $\left.\operatorname{Div}^{\tau}(Z / T)\right)$ the groups of divisors on $Z$ which are algebraically equivalent to zero and $\tau$-equivalent to zero, respectively, relative to $T$. Then $\operatorname{Div}^{0}(Z / T) \subseteq \operatorname{Div}^{\tau}(Z / T)$.

Relative algebraic equivalence and semifactoriality. Let $X / R$ be a proper flat semifactorial $R$-scheme. Suppose that the generic fiber $X_{K}$ is geometrically normal and geometrically connected. Its Picard variety $\mathrm{Pic}_{X_{K} / K \text {,red }}^{0}$ is then an abelian variety [FGA VI 1966, 3.2]. Let $A / R$ be its Néron model, and let $n$ be the exponent of the component group of the special fiber of $A$. In this situation, [Pepin 2011, Corollary 3.14] can be read as follows: for any divisor $D_{K}$ on $X_{K}$ which is algebraically equivalent to zero, there exists a divisor $\Delta$ on $X$ which is algebraically equivalent to zero relative to $R$ and whose generic fiber $\Delta_{K}$ is equal to $n D_{K}$. 
Definition 2.1.1. Let $X_{K}$ be a proper, geometrically normal and geometrically connected scheme over $K$. Let $X / R$ be a proper, flat, normal and semifactorial model of $X_{K}$ over $R$.

Consider $c_{K} \in Z_{0}^{0}\left(X_{K}\right)$ and $D_{K} \in \operatorname{Div}^{\tau}\left(X_{K}\right)$ with disjoint supports. Let $\bar{c}_{K}$ be the schematic closure of $c_{K}$ in $X$. Choose any $(n, \Delta) \in(\mathbb{Z} \backslash\{0\}) \times \operatorname{Div}^{\tau}(X / R)$ such that $\Delta_{K}=n D_{K}$. Then set

$$
\left[c_{K}, D_{K}\right]_{X}:=\frac{1}{n}\left(\bar{c}_{K} \cdot \Delta\right) \in \mathbb{Q} .
$$

This definition makes sense because $\frac{1}{n} \Delta \in \operatorname{Div}^{\tau}(X / R) \otimes_{\mathbb{Z}} \mathbb{Q}$ is uniquely determined by $D_{K}$, up to a rational multiple of the principal divisor $X_{k}$. Indeed, if $\left(n^{\prime}, \Delta^{\prime}\right)$ is another choice in Definition 2.1.1, then the divisor $n^{\prime} \Delta-n \Delta^{\prime}$ is $\tau$-equivalent to zero on $X$ and equal to zero on $X_{K}$. Thus, as $X$ is normal, this difference is a rational multiple of $X_{k}$ [Raynaud 1970, 6.4.13]. Now note that $\left(\bar{c}_{K} \cdot X_{k}\right)$ is equal to the degree of $c_{K}$, which is zero, so that $\frac{1}{n}\left(\bar{c}_{K} \cdot \Delta\right)=\frac{1}{n^{\prime}}\left(\bar{c}_{K} \cdot \Delta^{\prime}\right)$.

Next, one checks easily that the symbol $[\cdot, \cdot]_{X}$ is bilinear (in its definition domain). To prove that this pairing does not depend on the choice of $X$, we will use the following lemma.

We will denote by $(\cdot)_{*}$ and $(\cdot)^{*}$ the push-forward of cycles and the pull-back of divisors respectively; see [Fulton 1998, 20.1.3] and [Liu 2002, 7.1.29, 7.1.33, 7.1.34], respectively.

Lemma 2.1.2. Let $X$ and $X^{\prime}$ be integral schemes, proper over $R$. Let $\varphi: X \rightarrow X^{\prime}$ be an $R$-morphism. Let $c_{K} \in Z_{0}^{0}\left(X_{K}\right)$ and let $\bar{c}_{K}$ be its schematic closure in $X$. Let $\Delta^{\prime}$ be a divisor on $X^{\prime}$ whose support does not meet that of $\left(\varphi_{K}\right)_{*} c_{K}$. Then the following projection formula holds:

$$
\bar{c}_{K} \cdot \varphi^{*} \Delta^{\prime}=\varphi_{*} \bar{c}_{K} \cdot \Delta^{\prime}
$$

In particular, let $X$ and $X^{\prime}$ be proper, flat, normal and semifactorial schemes over $R$, with geometrically normal and geometrically connected generic fibers, so that $[\cdot, \cdot]_{X}$ and $[\cdot, \cdot]_{X^{\prime}}$ are defined. Let $\varphi: X \rightarrow X^{\prime}$ be an $R$-morphism. Let $c_{K} \in Z_{0}^{0}\left(X_{K}\right)$, and let $D_{K}^{\prime} \in \operatorname{Div}^{\tau}\left(X_{K}^{\prime}\right)$ whose support does not meet that of $\left(\varphi_{K}\right)_{*} c_{K}$. Then the following equality holds:

$$
\left[c_{K},\left(\varphi_{K}\right)^{*} D_{K}^{\prime}\right]_{X}=\left[\left(\varphi_{K}\right)_{*} c_{K}, D_{K}^{\prime}\right]_{X^{\prime}} .
$$

Proof. Let us first note that the divisors $\varphi^{*} \Delta^{\prime}$ (and $\left(\varphi_{K}\right)^{*} D_{K}^{\prime}$ ) are well-defined. Indeed, as $\varphi$ is proper, its image $Y$ is a closed subset of $X^{\prime}$. Endow $Y$ with its 
reduced scheme structure. As $X$ is reduced, $\varphi$ factors through $Y$ :

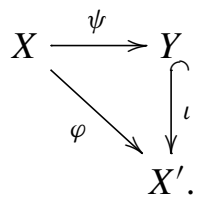

Now, by hypothesis, the support of $\Delta^{\prime}$ is disjoint from that of $\left(\varphi_{K}\right)_{*} c_{K}$. In particular, $Y$ is not contained in the support of $\Delta^{\prime}$. So the pullback $\iota^{*} \Delta^{\prime}$ is well-defined. Next, $X$ and $Y$ being integral and $\psi$ dominant, $\varphi^{*} \Delta^{\prime}:=\psi^{*}\left(\iota^{*} \Delta^{\prime}\right)$ is well-defined.

Let us now recall the proof of the projection formula $\bar{c}_{K} \cdot \varphi^{*} \Delta^{\prime}=\varphi_{*} \bar{c}_{K} \cdot \Delta^{\prime}$. Let $x_{K}$ be a closed point of the support of $c_{K}$, let $Z$ be its schematic closure in $X$, set $x_{k}:=Z \cap X_{k}$ and let $\widetilde{Z}$ be the normalization of $Z$. The reduced scheme $V:=\underset{\sim}{\varphi}(Z)$ is the schematic closure of $\varphi\left(x_{K}\right)$ and we have $\varphi\left(x_{k}\right)=V \cap X_{k}$. Denote by $\widetilde{V}$ the normalization of $V$. The morphism $\varphi$ induces a finite surjective morphism $Z \rightarrow V$, which in turn induces a finite surjective morphism $\widetilde{Z} \rightarrow \widetilde{V}$ ( $R$ is excellent). Let $f^{\prime}$ be a local equation of $\Delta^{\prime}$ at $\varphi\left(x_{k}\right)$. Suppose for example that $\left.f^{\prime}\right|_{\tilde{V}} \in \mathcal{O}(\tilde{V})$. The equality $\left(\varphi_{*} \bar{c}_{K} \cdot \Delta^{\prime}\right)_{x_{k}}=\left(\bar{c}_{K} \cdot \varphi^{*} \Delta^{\prime}\right)_{x_{k}}$ to be proved can be written as

$$
[K(Z): K(V)] \cdot \operatorname{length}\left(\mathcal{O}(\widetilde{V}) /\left(f^{\prime}\right)\right)=\operatorname{length}\left(\mathcal{O}(\widetilde{Z}) /\left(\varphi^{*} f^{\prime}\right)\right) .
$$

But $[K(Z): K(V)]$ is equal to the ramification index of the discrete valuation rings extension $\mathcal{O}(\tilde{V}) \rightarrow \mathcal{O}(\widetilde{Z})$. Consequently, the above formula is true.

Now, when the pairings $[\cdot, \cdot]_{X}$ and $[\cdot, \cdot]_{X^{\prime}}$ are defined, the projection formula can be written as the equality $\left[c_{K},\left(\varphi_{K}\right)^{*} D_{K}^{\prime}\right]_{X}=\left[\left(\varphi_{K}\right)_{*} c_{K}, D_{K}^{\prime}\right]_{X^{\prime}}$. Indeed, let $\Delta^{\prime}$ be a divisor which is $\tau$-equivalent to zero on $X^{\prime}$ and let $n^{\prime}$ be a nonzero integer such that $\left(\Delta^{\prime}\right)_{K}=n^{\prime} D_{K}^{\prime}$. The direct image $\varphi_{*} \bar{c}_{K}$ of the schematic closure of $c_{K}$ coincides with the schematic closure of $\left(\varphi_{K}\right)_{*} c_{K}$. Thus, by definition,

$$
n^{\prime}\left[\left(\varphi_{K}\right)_{*} c_{K}, D_{K}^{\prime}\right]_{X^{\prime}}=\varphi_{*} \bar{c}_{K} \cdot \Delta^{\prime} .
$$

The divisor $\varphi^{*} \Delta^{\prime}$ is $\tau$-equivalent to zero on $X$, and satisfies $\left(\varphi^{*} \Delta^{\prime}\right)_{K}=n^{\prime}\left(\varphi_{K}\right)^{*} D_{K}^{\prime}$. Hence, by definition,

$$
n^{\prime}\left[c_{K},\left(\varphi_{K}\right)^{*} D_{K}^{\prime}\right]_{X}=\bar{c}_{K} \cdot \varphi^{*} \Delta^{\prime} .
$$

In the situation of Definition 2.1.1, let $X^{\prime}$ be another proper flat normal semifactorial $R$-model of $X_{K}$. Consider the graph $\Gamma$ of the rational map $X \rightarrow X^{\prime}$ induced by the identity on the generic fibers. By definition, this is the schematic closure of the graph of the identity morphism $X_{K} \rightarrow X_{K}^{\prime}$ in $X \times_{R} X^{\prime}$. In particular, this is a closed subscheme of $X \times{ }_{R} X^{\prime}$, proper and flat over $R$, with generic fiber isomorphic to $X_{K}$. Applying [Pepin 2011, Theorem 2.6], we can find an $R$-scheme $\widetilde{X}$ which is proper flat normal and semifactorial, together with an $R$-morphism $\widetilde{X} \rightarrow \Gamma$ which 
is an isomorphism on the generic fibers. Composing with the two projections from $X \times{ }_{R} X^{\prime}$ to $X$ and $X^{\prime}$, we get arrows

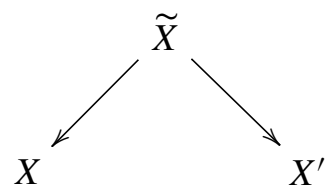

which are isomorphisms on the generic fibers. Now, Lemma 2.1.2 shows that the pairings $[\cdot, \cdot]_{X}$ and $[\cdot, \cdot]_{X^{\prime}}$ both coincide with $[\cdot, \cdot]_{\tilde{X}}$. In conclusion, the pairing $[\cdot, \cdot]_{X}$ depends only on $X_{K}$, and not on the choice of $X$.

Let us summarize the above considerations:

Proposition 2.1.3. Let $X_{K}$ be a proper, geometrically normal and geometrically connected scheme over $K$. There exists a pairing

$$
[\cdot, \cdot]: Z_{0}^{0}\left(X_{K}\right) \times \operatorname{Div}^{\tau}\left(X_{K}\right) \rightarrow \mathbb{Q},
$$

defined for the pairs $\left(c_{K}, D_{K}\right)$ such that the supports of $c_{K}$ and $D_{K}$ are disjoint, and which can be computed as follows.

Let $X / R$ be any proper flat normal and semifactorial model of $X_{K}$ over $R$. Let $\bar{c}_{K}$ be the schematic closure of $c_{K}$ in $X$. Choose $(n, \Delta) \in(\mathbb{Z} \backslash\{0\}) \times \operatorname{Div}^{\tau}(X / R)$ such that $\Delta_{K}=n D_{K}$. Then we have

$$
\left[c_{K}, D_{K}\right]=\frac{1}{n}\left(\bar{c}_{K} \cdot \Delta\right) \in \mathbb{Q} .
$$

2.2. Comparison with Néron's pairing. As before, let $R$ be a complete discrete valuation ring with fraction field $K$ and algebraically closed residue field $k$. Let $X_{K}$ be a proper smooth and geometrically connected scheme over $K$. Let $v$ be the normalized valuation on $K$, which maps any uniformizing element of $R$ to $1 \in \mathbb{Z}$. We fix an algebraic closure $\bar{K}$ of $K$, and we still denote by $v$ the unique valuation on $\bar{K}$ extending $v$. Néron attached to $X_{K}$ a pairing $\langle\cdot, \cdot\rangle$ with respect to the valuation $v$ [Néron 1965, II 9.3]. This is a pairing

$$
\langle\cdot, \cdot\rangle: Z_{0}^{0}\left(X_{K}\right) \times \operatorname{Div}^{\tau}\left(X_{K}\right) \rightarrow \mathbb{R},
$$

defined for $\left(c_{K}, D_{K}\right)$ when the supports of $c_{K}$ and $D_{K}$ are disjoint (the definition of Néron's pairing is briefly reviewed at the beginning of the proof of Theorem 2.2.1). Actually, Néron considers the subgroup $\operatorname{Div}^{0}\left(X_{K}\right) \subseteq \operatorname{Div}^{\tau}\left(X_{K}\right)$ to consist of divisors which are algebraically equivalent to zero on $X_{K}$. However, the group $(\mathbb{R},+)$ being divisible, the real number $\left\langle c_{K}, D_{K}\right\rangle$ is naturally defined when $D_{K}$ is only $\tau$-equivalent to zero. Néron shows in [loc. cit., III 4.2] that the pairing takes values in $\mathbb{Q}$. This fact will be recovered and made more precise below (Corollary 2.2.2). 
Our goal in this subsection is to prove the following common generalization of Néron [1965, III 4.1], Gross [1986], Hriljac [1985], Lang [1988, III 5.2] and BoschLorenzini [2002, 4.3], over a complete discrete valuation ring $R$ with algebraically closed residue field $k$ and fraction field $K$.

Theorem 2.2.1. For every proper, smooth and geometrically connected scheme over $K$, the pairing $[\cdot, \cdot]$ defined in Proposition 2.1.3 coincides with Néron's pairing $\langle\cdot, \cdot\rangle$ defined in [Néron 1965, II.9, Theorem 3].

In particular, the pairing $[\cdot, \cdot]$ generalizes Néron's pairing to $K$-schemes which are proper geometrically normal and geometrically connected, but not necessarily smooth.

Before proving the theorem, let us note a consequence of Proposition 2.1.3.

Corollary 2.2.2. Let $X_{K}$ be a proper, geometrically normal and geometrically connected scheme over $K$. Let $n$ be the exponent of the component group of the special fiber of the Néron model of the Picard variety $A_{K}=\mathrm{Pic}_{X_{K} / K \text {,red. Then }}^{0}$ Néron's pairing on $Z_{0}^{0}\left(X_{K}\right) \times \operatorname{Div}^{0}\left(X_{K}\right)$ takes values in $(1 / n) \mathbb{Z}$.

Proof. As recalled before Definition 2.1.1, the exponent $n$ has the following property: for any $D_{K} \in \operatorname{Div}^{0}\left(X_{K}\right)$ and any proper flat normal and semifactorial model $X$ of $X_{K}$, there exists $\Delta \in \operatorname{Div}^{0}(X / R)$ such that $\Delta_{K}=n D_{K}$. In particular, for any $D_{K} \in \operatorname{Div}^{0}\left(X_{K}\right)$, we can choose this integer $n$, together with a divisor $\Delta \in \operatorname{Div}^{0}(X / R)$, to compute

$$
\left[c_{K}, D_{K}\right]=\frac{1}{n}\left(\bar{c}_{K} \cdot \Delta\right) \in \frac{1}{n} \mathbb{Z} .
$$

Now Theorem 2.2.1 asserts that $\left\langle c_{K}, D_{K}\right\rangle=\left[c_{K}, D_{K}\right]$.

Corollary 2.2.2 provides a refinement of [Néron 1965, III 4.2]. More precisely, Néron shows that the pairing

$$
\langle\cdot, \cdot\rangle: Z_{0}^{0}\left(X_{K}\right) \times \operatorname{Div}^{0}\left(X_{K}\right) \rightarrow \mathbb{R}
$$

takes values in $\left(1 / 2 n^{\prime} a b\right) \mathbb{Z}$, where $n^{\prime}, a$ and $b$ are defined as follows. The integer $n^{\prime}$ is the exponent of the component group of the special fiber of the Neron model of the Albanese variety $A_{K}^{\prime}$ of $X_{K}$. Conjecturally, $n^{\prime}$ is equal to $n$; see Section 3.1. Next, $a$ is the smallest positive integer such that there exists a map $h: X_{K} \rightarrow A_{K}^{\prime}$ from $X_{K}$ to its Albanese variety, with the property that for any divisor $D_{K} \in \operatorname{Div}^{0}\left(X_{K}\right)$, there exists a divisor $W_{K} \in \operatorname{Div}^{0}\left(A_{K}^{\prime}\right)$ such that $h^{*} W_{K}$ is linearly equivalent to $a D_{K}$. We can have $a>1$ if $X_{K}(K)$ is empty. Finally, $b$ is the smallest degree of a polarization of the Albanese variety $A_{K}^{\prime}$.

In [Mazur and Tate 1983, (1.5) and (2.3); Lang 1983, 11.5.1-11.5.2], it is proved that $\left\langle c_{K}, D_{K}\right\rangle$ belongs to $\left(1 / n^{\prime}\right) \mathbb{Z}$ when $X_{K}$ is an abelian variety and if $c_{K}$ is supported on rational points. This statement is also a consequence of [Bosch and 
Lorenzini 2002, 4.4]. Moreover, note that Néron's pairing can take the value $1 / n$, for instance when $X_{K}$ is an elliptic curve; see [loc. cit., Example 5.8].

Let us go back to Theorem 2.2.1. To prove the theorem, we will use the characterization of Néron's pairing given in [Lang 1983, 11.3.2] and that we recall now.

An element $c_{K}$ of $Z_{0}^{0}\left(X_{K}\right)$ can be written uniquely as a difference of two positive 0 -cycles with disjoint supports: $c_{K}=c_{K}^{+}-c_{K}^{-}$. Denoting by deg the degree of a 0 -cycle, let us set

$$
\operatorname{deg}^{+} c_{K}:=\operatorname{deg}\left(c_{K}^{+}\right)=\operatorname{deg}\left(c_{K}^{-}\right) \geq 0 .
$$

Lemma 2.2.3 [Lang 1983, 11.3.2]. Suppose that for each projective smooth and geometrically connected scheme $X_{K}$ over $K$, we are given a bilinear pairing

$$
\begin{aligned}
Z_{0}^{0}\left(X_{K}\right) \times \operatorname{Div}^{0}\left(X_{K}\right) & \rightarrow \mathbb{R} \\
\left(c_{K}, D_{K}\right) & \mapsto \delta\left(c_{K}, D_{K}\right)
\end{aligned}
$$

such that the following properties are true:

(1) If $D_{K}$ is a principal divisor on $X_{K}$, then $\delta\left(c_{K}, D_{K}\right)=0$.

(2) Let $\varphi_{K}: X_{K} \rightarrow X_{K}^{\prime}$ be a $K$-morphism. For all $c_{K} \in Z_{0}^{0}\left(X_{K}\right)$, and for all $D_{K}^{\prime} \in \operatorname{Div}^{0}\left(X_{K}^{\prime}\right)$ whose support does not meet that of the 0 -cycle $\left(\varphi_{K}\right)_{*} c_{K}$, the following equality holds:

$$
\delta\left(c_{K},\left(\varphi_{K}\right)^{*} D_{K}^{\prime}\right)=\delta\left(\left(\varphi_{K}\right)_{*} c_{K}, D_{K}^{\prime}\right) .
$$

(3) For $D_{K} \in \operatorname{Div}^{0}\left(X_{K}\right)$ fixed and $\operatorname{deg}^{+} c_{K}$ bounded, the values $\delta\left(c_{K}, D_{K}\right)$ are bounded.

Then $\delta\left(c_{K}, D_{K}\right)=0$ for all $c_{K}, D_{K}$ and $X_{K}$.

Remark 2.2.4. In the statement of [Lang 1983, 11.3.2], one reads "projective variety $V$ over $K$ " instead of "projective smooth and geometrically connected scheme $X_{K}$ over $K$ ". According to the general conventions of [loc. cit., page 21 ], a "variety over $K$ " is a "geometrically integral scheme of finite type over $K$ ". However, the given proof of [loc. cit., 11.3.2] works if and only if the Albanese variety of each $V$ is an abelian variety. The latter is true, for example, if each $V$ is geometrically normal, or if each $V$ is smooth. For our purposes, namely the proof of Theorem 2.2.1, we need the version of the lemma where all the $V$ are smooth.

Proof of Theorem 2.2.1. Starting from the existence of Néron functions on a proper smooth and geometrically connected $K$-scheme $X_{K}$ [Néron 1965, II 8.2], let us recall the definition of Néron's pairing. Let

$$
c_{K}=\sum_{i} n_{i}\left[x_{K, i}\right] \in Z_{0}^{0}\left(X_{K}\right)
$$


and take $D_{K} \in \operatorname{Div}^{0}\left(X_{K}\right)$ whose support $\operatorname{Supp}\left(D_{K}\right)$ does not contain any of the $x_{K, i}$. Let $\lambda_{D_{K}}:\left(X_{K}-\operatorname{Supp}\left(D_{K}\right)\right)(\bar{K}) \rightarrow \mathbb{R}$ be a Néron function associated to $D_{K}$. For each $i$, the scheme $x_{K, i} \otimes_{K} \bar{K}$ is supported on some $\bar{K}$-points $x_{\bar{K}, j_{i}}, j_{i}=1, \ldots, s_{i}$, where $s_{i}$ is the separable degree of $K\left(x_{K, i}\right) / K$. Denoting by $l_{i}$ the inseparable degree of $K\left(x_{K, i}\right) / K$, then

$$
\lambda_{D_{K}}\left(x_{K, i}\right):=\sum_{j_{i}=1}^{s_{i}} l_{i} \lambda_{D_{K}}\left(x_{\bar{K}, j_{i}}\right) \quad \text { and } \quad\left\langle c_{K}, D_{K}\right\rangle:=\sum_{i} n_{i} \lambda_{D_{K}}\left(x_{K, i}\right) .
$$

The real number $\left\langle c_{K}, D_{K}\right\rangle$ is well-defined because $\lambda_{D_{K}}$ is unique up to constant and $c_{K}$ has degree zero.

Comparison of the pairings for a principal divisor $D_{K}$. Let us keep the previous notation, and suppose that $D_{K}=\operatorname{div}_{X_{K}} f$ for a nonzero $f \in K\left(X_{K}\right)$. Let $z \in$ $\left(X_{K}-\operatorname{Supp}\left(\operatorname{div}_{X_{K}} f\right)\right)(\bar{K})$, mapping to a closed point $x_{K} \in X_{K}$. The evaluation of $f$ at $z$ is defined by the pull-back $z^{*}: \mathscr{O}_{X_{K}, x_{K}} \rightarrow \bar{K}$, that is, $f(z):=z^{*} f$. The formula $\lambda_{f}(z)=v(f(z))$ then defines a Néron function for the $\operatorname{divisor} \operatorname{div}_{X_{K}} f$.

Fix an $i$. There is a 1-1 correspondence between the $x_{\bar{K}, j_{i}}$ and the $K$-embeddings of the residue field extension $K\left(x_{K, i}\right) / K$ into $\bar{K} / K$. By pulling back the valuation $v$, each of these embeddings induces a valuation on $K\left(x_{K, i}\right)$. However, as $R$ is complete, these valuations are equal to the unique valuation on $K\left(x_{K, i}\right)$ which extends the normalized valuation on $K$, and that we can also denote by $v$. Consequently,

$$
\lambda_{f}\left(x_{K, i}\right)=\sum_{j_{i}=1}^{s_{i}} l_{i} v\left(f\left(x_{K, i}\right)\right)=\left[K\left(x_{K, i}\right): K\right] v\left(f\left(x_{K, i}\right)\right)
$$

where $f\left(x_{K, i}\right)$ is the image of $f$ by the canonical surjection $\mathscr{O}_{X_{K}, x_{K, i}} \rightarrow K\left(x_{K, i}\right)$.

Now, take the schematic closure $Z_{i}$ of $x_{K, i}$ in $X$, denote by $\widetilde{Z}_{i}$ its normalization and set $x_{k, i}:=X_{k} \cap Z_{i}$. The ring $\mathcal{O}\left(\widetilde{Z}_{i}\right)$ is a discrete valuation ring with fraction field $K\left(x_{K, i}\right)$. So it is precisely the valuation ring of $v$ in $K\left(x_{K, i}\right)$. As $k$ is algebraically closed, its ramification index over $R$ is equal to $\left[K\left(x_{K, i}\right): K\right]$. From this observation, we get

$$
v\left(f\left(x_{K, i}\right)\right)=\left\{\begin{array}{cl}
1 /\left[K\left(x_{K, i}\right): K\right] \text { length }_{\mathscr{O}\left(\widetilde{Z}_{i}\right)}\left(\mathcal{O}\left(\widetilde{Z}_{i}\right) /(f)\right) & \text { if }\left.f\right|_{\tilde{Z}_{i}} \in \mathcal{O}\left(\widetilde{Z}_{i}\right), \\
-1 /\left[K\left(x_{K, i}\right): K\right] \text { length }_{\mathscr{O}\left(\widetilde{Z}_{i}\right)}\left(\mathcal{O}\left(\widetilde{Z}_{i}\right) /\left(f^{-1}\right)\right) & \text { otherwise. }
\end{array}\right.
$$

We have thus obtained $\left[K\left(x_{K, i}\right): K\right] v\left(f\left(x_{K, i}\right)\right)=\left(\bar{c}_{K} \cdot \operatorname{div}_{X} f\right)_{x_{k, i}}$ (recall the beginning of Section 2.1). But $\operatorname{div}_{X} f$ is a divisor on $X$ which is $\tau$-equivalent to zero and extends $\operatorname{div}_{X_{K}} f$. The desired equality $\left\langle c_{K}, \operatorname{div}_{X_{K}} f\right\rangle=\left[c_{K}, \operatorname{div}_{X_{K}} f\right]$ follows.

Functoriality of the pairing $[\cdot, \cdot]$. Let $\varphi_{K}: X_{K} \rightarrow X_{K}^{\prime}$ be a $K$-morphism of proper smooth and geometrically connected schemes over $K$. Let us show that for all $c_{K} \in Z_{0}^{0}\left(X_{K}\right)$, and for all $D_{K}^{\prime} \in \operatorname{Div}^{\tau}\left(X_{K}^{\prime}\right)$ whose support does not meet that of the 
0 -cycle $\left(\varphi_{K}\right)_{*} c_{K}$, the following equality holds

$$
\left[c_{K},\left(\varphi_{K}\right)^{*} D_{K}^{\prime}\right]=\left[\left(\varphi_{K}\right)_{*} c_{K}, D_{K}^{\prime}\right] .
$$

Let $X / R$ (resp. $X^{\prime} / R$ ) be a proper flat normal semifactorial model of $X_{K}$ (resp. $\left.X_{K}^{\prime}\right)$. Consider the graph $\Gamma$ of the rational map $X \rightarrow X^{\prime}$ defined by $\varphi_{K}$. Applying Theorem 2.6 of [Pepin 2011] to $\Gamma$, we obtain a proper flat normal semifactorial $\widetilde{X} / R$ and $R$-morphisms

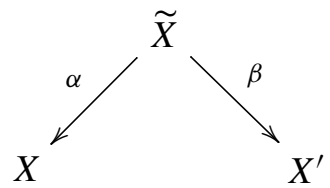

such that on the generic fibers, $\alpha$ is an isomorphism and $\beta$ coincides with $\varphi_{K}$. In particular, the pairing $[\cdot, \cdot]$ for $X_{K}$ can be computed on $\tilde{X}$, and the desired functoriality follows from Lemma 2.1.2 applied to $\beta$.

The pairing $\delta(\cdot, \cdot)$. At this point, we recall that for any proper smooth and geometrically connected scheme $X_{K}$ over $K$, there exists a nonzero integer $a$ and a map $X_{K} \rightarrow A_{K}^{\prime}$ from $X_{K}$ to its Albanese variety, with the property that for any divisor $D_{K} \in \operatorname{Div}^{\tau}\left(X_{K}\right)$, there exists a divisor $W_{K} \in \operatorname{Div}^{0}\left(A_{K}^{\prime}\right)$ such that $h^{*} W_{K}$ is welldefined and linearly equivalent to $a D_{K}$ [Néron 1965, II 2.1]. Let $c_{K} \in Z_{0}^{0}\left(X_{K}\right)$ and $D_{K} \in \operatorname{Div}^{\tau}\left(X_{K}\right)$ with disjoint supports. Keep the previous notation. After moving $W_{K}$ on the projective smooth scheme $A_{K}^{\prime}$ if necessary (see [Liu 2002, 9.1.11], for example), we can assume that the support of $h^{*} W_{K}$ does not meet that of $c_{K}$. Then, using the functoriality of $[\cdot, \cdot]$, we can write

$$
a\left[c_{K}, D_{K}\right]=\left[c_{K}, h^{*} W_{K}\right]+\left[c_{K}, \operatorname{div}_{X_{K}} f\right]=\left[h_{*} c_{K}, W_{K}\right]+\left[c_{K}, \operatorname{div}_{X_{K}} f\right]
$$

for some nonzero $f \in K\left(X_{K}\right)$. By definition, Néron's pairing has the same functoriality property as $[\cdot, \cdot]$. And we have seen that both pairings coincide for principal divisors. Consequently, as $A_{K}^{\prime}$ is projective smooth geometrically connected over $K$, Theorem 2.2.1 is proved if we know that both pairings coincide on such schemes. So, until the end of the proof, we will only consider the pairings for projective smooth geometrically connected schemes. Furthermore, by $\mathbb{Z}$-linearity, we can only consider divisors which are algebraically equivalent to zero.

Now, both $[\cdot, \cdot]$ and $\langle\cdot, \cdot\rangle$ are bilinear in their definition domain, and they coincide for principal divisors. Using a moving lemma on the projective smooth scheme $X_{K}$, we see that

$$
\delta\left(c_{K}, D_{K}\right):=\left\langle c_{K}, D_{K}\right\rangle-\left[c_{K}, D_{K}\right]
$$

is well-defined on the whole product $Z_{0}^{0}\left(X_{K}\right) \times \operatorname{Div}^{0}\left(X_{K}\right)$. And conditions (1) and (2) of Lemma 2.2.3 are satisfied by $\delta$. 
Condition (3) of Lemma 2.2.3 is satisfied by $\delta(\cdot, \cdot)$. Denote by $\bar{R}$ the valuation ring of $v$ in $\bar{K}$.

Fix $D_{K} \in \operatorname{Div}^{0}\left(X_{K}\right)$. Let $(n, \Delta) \in(\mathbb{Z} \backslash\{0\}) \times \operatorname{Div}^{\tau}\left(X_{K}\right)$ satisfying $\Delta_{K}=n D_{K}$. Represent the divisor $\Delta$ by a family $\left(U_{t}, g_{t}\right)_{t=1, \ldots, m}$, where the $U_{t}$ are affine open subsets of $X$ and the $g_{t}$ are rational functions on $X$. Let $E_{t}$ be the set of $\bar{K}$ points of $X_{K}$ which extend to $\bar{R}$-points of $U_{t}$. As $X$ is proper over $R$, we see that $X(\bar{K})=\cup_{t=1}^{m} E_{t}$. The family $\left(U_{t, K}, g_{t}\right)_{t=1, \ldots, m}$ represents the divisor $n D_{K}$ on $X_{K}$. Let us choose a Néron function $\lambda_{n D_{K}}$ on $X_{K}$. By definition, we can find some $v$-continuous locally bounded functions $\alpha_{t}: U_{t, K}(\bar{K}) \rightarrow \mathbb{R}$ such that

$$
\lambda_{n D_{K}}(z)=v\left(g_{t}(z)\right)+\alpha_{t}(z)
$$

for all $z \in\left(U_{t, K}-\operatorname{Supp}\left(D_{K}\right)\right)(\bar{K})$. As $E_{t}$ is bounded in $U_{t}(\bar{K})$ (by construction), the function $\alpha_{t}$ is bounded on $E_{t}$.

Let $c_{K}=\sum_{i} n_{i}\left[x_{K, i}\right] \in Z_{0}^{0}\left(X_{K}\right)$ whose support does not meet that of $D_{K}$. Fix an $i$, let $Z_{i}$ be the schematic closure of $x_{K, i}$ in $X$, set $x_{k, i}:=X_{k} \cap Z_{i}$ and let $t_{i}$ be such that $Z_{i} \subset U_{t_{i}}$. The same local computation as in the case of a principal divisor shows that

$$
\left(\bar{c}_{K} \cdot \Delta\right)_{x_{k, i}}=\left[K\left(x_{K, i}\right): K\right] v\left(g_{t_{i}}\left(x_{K, i}\right)\right)=\sum_{j_{i}=1}^{s_{i}} l_{i} v\left(g_{t_{i}}\left(x_{K, i}\right)\right) .
$$

On the other hand, keeping the same notation as in the beginning of the proof,

$$
\left\langle c_{K}, n D_{K}\right\rangle=\sum_{i} n_{i} \sum_{j_{i}=1}^{s_{i}} l_{i} \lambda_{n D_{K}}\left(x_{\bar{K}, j_{i}}\right) .
$$

Consequently,

$$
n \delta\left(c_{K}, D_{K}\right)=\sum_{i} n_{i} \sum_{j_{i}=1}^{s_{i}} l_{i} \alpha_{t_{i}}\left(x_{\bar{K}, j_{i}}\right) .
$$

By construction, the $\bar{K}$-point $x_{\bar{K}, j_{i}}$ of $X_{K}$ belongs to $E_{t_{i}}$. Denoting by $|\cdot|$ the usual absolute value on $\mathbb{R}$, and setting

we obtain

$$
B:=\max _{t=1, \ldots, m}\left(\sup _{E_{t}}\left|\alpha_{t}\right|\right) \in \mathbb{R}
$$

$$
\left|\delta\left(c_{K}, D_{K}\right)\right| \leq \frac{1}{|n|} \sum_{i}\left|n_{i}\right|\left[K\left(x_{K, i}\right): K\right] B=\frac{2 B}{|n|} \operatorname{deg}^{+} c_{K} .
$$

As the divisor $D_{K}$ is fixed, the numbers $n$ and $B$ are fixed, and so the right-hand side of the above inequality is bounded if $\operatorname{deg}^{+} c_{K}$ is.

Let us note the following properties of the pairing $[\cdot, \cdot]$, and consequently of Néron's pairing. 
Proposition 2.2.5. Let $X_{K}$ be a proper, geometrically normal and geometrically connected scheme over $K$. Let $c_{K} \in Z_{0}^{0}\left(X_{K}\right)$ and let $D_{K} \in \operatorname{Div}^{\tau}\left(X_{K}\right)$ with disjoint supports. If $c_{K}$ or $D_{K}$ is rationally equivalent to zero, then $\left[c_{K}, D_{K}\right] \in \mathbb{Z}$.

Proof. The case where $D_{K}$ is rationally equivalent to zero follows directly from the definition of $[\cdot, \cdot]$ : if $D_{K}=\operatorname{div}_{K} f$ with $f \in K\left(X_{K}\right) \backslash\{0\}$, then $\left[c_{K}, \operatorname{div}_{K} f\right]=$ $\left(c_{K} \cdot \operatorname{div} f\right) \in \mathbb{Z}$.

Let us now suppose that $c_{K}$ is rationally equivalent to zero. As [, $\left.D_{K}\right]$ is $\mathbb{Z}$-linear, we have to show that if $c_{K}=\left(\varphi_{K}\right)_{*} \operatorname{div}_{C_{K}} f$ for some $K$-morphism

$$
\varphi_{K}: C_{K} \rightarrow X_{K}
$$

from a proper normal connected curve $C_{K}$ to $X_{K}$, and some nonzero $f \in K\left(C_{K}\right)$, then

$$
\left[c_{K}, D_{K}\right] \in \mathbb{Z} .
$$

As $R$ is excellent, there exists a proper flat regular model $C / R$ of $C_{K}$. On the other hand, let us consider a proper flat normal semifactorial model $X / R$ of $X_{K}$. After replacing $C$ by a desingularization of the graph of the rational map $C \rightarrow X$ induced by $\varphi_{K}$, we can suppose that $\varphi_{K}$ extends to an $R$-morphism $\varphi: C \rightarrow X$. If $\Delta$ is a divisor on $X$ which is $\tau$-equivalent to zero and such that $\Delta_{K}=n D_{K}$ for some integer $n \neq 0$, then

$$
\left[c_{K}, D_{K}\right]:=\frac{1}{n}\left(\overline{\left(\varphi_{K}\right)_{*} \operatorname{div}_{C_{K}} f} \cdot \Delta\right)=\frac{1}{n}\left(\overline{\operatorname{div}_{C_{K}} f} \cdot \varphi^{*} \Delta\right)
$$

by the projection formula (Lemma 2.1.2). Let us write

$$
\operatorname{div}_{C} f=\overline{\operatorname{div}_{C_{K}} f}-V \quad \text { and } \quad \varphi^{*} \Delta=\overline{\left(\varphi_{K}\right)^{*} \Delta_{K}}-W
$$

for some vertical divisors $V$ and $W$ on $C / R$. Denote by $\Gamma_{1}, \ldots, \Gamma_{v}$ the reduced irreducible components of $C_{k}$, by $M$ the intersection matrix associated to $C_{k}$ (as defined in the introduction), and by $\rho: \operatorname{Pic}(C) \rightarrow \mathbb{Z}^{v}$ the degree homomorphism $(E) \mapsto\left(E \cdot \Gamma_{i}\right)_{i=1, \ldots, \nu}$. Following [Bosch et al. 1990, 9.2/13], the divisor $E$ on the $R$-curve $C$ is algebraically equivalent to zero if and only if $(E)$ belongs to the kernel of $\rho$. Therefore the $\tau$-equivalence relation and the algebraic equivalence relation on $C / R$ are the same, and the linear equivalence classes of $\varphi^{*} \Delta$ and $\operatorname{div}_{C} f$ belongs to the kernel of $\rho$. Thus we get:

$$
\rho\left(\overline{\operatorname{div}_{C_{K}} f}\right)=\rho(V)=M V \quad \text { and } \quad \rho\left(\overline{\left(\varphi_{K}\right)^{*} \Delta_{K}}\right)=\rho(W)=M W,
$$

where we have identified a vertical divisor on $C / R$ with an element of $\mathbb{Z}^{v}$. Next, we use that the matrix $M$ is symmetric to obtain

$$
\left(\overline{\operatorname{div}_{C_{K}} f} \cdot W\right)={ }^{t} W \rho\left(\overline{\operatorname{div}_{C_{K}} f}\right)={ }^{t} W M V={ }^{t} V M W=\left(\overline{\left(\varphi_{K}\right)^{*} \Delta_{K}} \cdot V\right) .
$$


Then it follows that

$$
\left[c_{K}, D_{K}\right]=\frac{1}{n}\left(\overline{\left(\varphi_{K}\right)^{*} \Delta_{K}} \cdot \operatorname{div}_{C} f\right)=\left(\overline{\left(\varphi_{K}\right)^{*} D_{K}} \cdot \operatorname{div}_{C} f\right) \in \mathbb{Z} .
$$

Remark 2.2.6. Let us keep the notation of the proof of 2.2.5. If the curve $C_{K}$ is geometrically normal and geometrically connected, the pairing $[\cdot, \cdot]$ is defined on $C_{K}$ and

$$
\left(\overline{\left(\varphi_{K}\right)^{*} D_{K}} \cdot \operatorname{div}_{C} f\right)=\left[\left(\varphi_{K}\right)^{*} D_{K}, \operatorname{div}_{C_{K}} f\right] .
$$

In other words, in this case, the proof consists in using the functoriality of the pairing $[\cdot, \cdot]$, then showing that it is symmetric for curves, and finally applying the definition of the pairing for a principal divisor. The symmetry property of Néron's pairing $\langle\cdot, \cdot\rangle$ for such a curve is well-known: for example see [Lang 1983, 11.3.6 and 11.3.7]. But here, there is no reason for the curve $C_{K}$ coming from the rational equivalence relation to satisfy the above geometric hypotheses. So we could not use directly the properties of the pairing $\langle\cdot, \cdot\rangle$. However, over an excellent discrete valuation ring, there is no need of these geometric hypotheses on $C_{K}$ for the existence of the regular model $C / R$. So we have been able to prove the proposition for the pairing $[\cdot, \cdot]$, and thus also for Néron's pairing $\langle\cdot, \cdot\rangle$ thanks to Theorem 2.2.1.

\section{Duality and algebraic equivalence for models of abelian varieties}

3.1. Grothendieck's duality for Néron models. Let us recall here Grothendieck's duality theory for Néron models of abelian varieties, as developed in [SGA 7 I 1972, VII, VIII, IX].

Let $R$ be a discrete valuation ring with perfect residue field $k$ and fraction field $K$. Let $A_{K}$ be an abelian variety over $K$, with dual $A_{K}^{\prime}$. Let $A / R, A^{\prime} / R$ be the Néron models of $A_{K}, A_{K}^{\prime}$, and $\Phi_{A}, \Phi_{A^{\prime}}$ be the étale $k$-group schemes of connected components of the special fibers $A_{k}, A_{k}^{\prime}$.

By definition, the abelian variety $A_{K}^{\prime}$ represents the identity component $\mathrm{Pic}_{A_{K} / K}^{0}$ of the Picard functor of $A_{K}$, and the canonical isomorphism $A_{K}^{\prime}=\mathrm{Pic}_{A_{K} / K}^{0}$ is given by the Poincaré sheaf $\mathscr{P}_{K}$ on $A_{K} \times_{K} A_{K}^{\prime}$ birigidified along the unit sections of $A_{K}$ and $A_{K}^{\prime}$. Now, this sheaf is canonically endowed with the structure of a biextension of $\left(A_{K}, A_{K}^{\prime}\right)$ by $\mathbb{G}_{m, K}$ [loc. cit., VII 2.9.5]. Then the duality theory for Néron models is to understand how this biextension extends at the level of Néron models. For this, Grothendieck attached to $\mathscr{P}_{K}$ a canonical pairing

$$
\langle\cdot, \cdot\rangle: \Phi_{A} \times_{k} \Phi_{A^{\prime}} \rightarrow \mathbb{Q} / \mathbb{Z}
$$

which measures the obstruction to extending $\mathscr{P}_{K}$ as a biextension of $\left(A, A^{\prime}\right)$ by $\mathbb{G}_{m, R}$. The duality statement is: this pairing is a perfect duality [loc. cit., IX 1.3]. As mentioned in the introduction, it has been proved in various situations, including the 
semistable case [SGA 7 I 1972, IX 11.4; Werner 1997] and the mixed characteristic case [Bégueri 1980]. In general, the duality statement remains a conjecture.

3.2. Duality and Picard functor. Keep the notation of the previous subsection. By [Pepin 2011, Corollary 2.23], it is always possible to find an $R$-compactification of $A$, that is, an open $R$-immersion of $A$ into a proper $R$-scheme $\bar{A}$ with dense image, such that $\bar{A} / R$ is flat, $\bar{A}$ is normal and the canonical map $\operatorname{Pic}(\bar{A}) \rightarrow \operatorname{Pic}(A)$ is surjective. Note that, in particular, $\bar{A} / R$ is semifactorial: the map $\operatorname{Pic}(A) \rightarrow \operatorname{Pic}\left(A_{K}\right)$ is surjective because $A$ is regular, so that $\operatorname{Pic}(\bar{A}) \rightarrow \operatorname{Pic}\left(A_{K}\right)$ is surjective by composition. As $\bar{A} / R$ is proper, it makes sense to consider the notion of algebraic equivalence on $\bar{A}$ relative to $R$ using the identity component of the Picard functor $\operatorname{Pic}_{\bar{A} / R}$, as defined in Section 2.1. Our goal in this section is to understand the duality from the point of view of algebraic equivalence, starting from the canonical isomorphism $A_{K}^{\prime}=\mathrm{Pic}_{A_{K} / K}^{0}$. To do this, we need the following notions.

$\mathbb{Q}$-divisors and relative $\tau$-equivalence. Let $Z$ be a normal locally noetherian scheme, so that the canonical homomorphism from the group of divisors on $Z$ into that of 1-codimensional cycles is injective [EGA IV ${ }_{4}$ 1967, 21.6.9(i)]. A 1-codimensional cycle $C$ on $Z$ is said to be a $\mathbb{Q}$-divisor if there exists $n \in \mathbb{Z} \backslash\{0\}$ such that $n C$ is a divisor.

Let $Z \rightarrow T$ be a proper morphism of schemes, with $Z$ locally noetherian and normal. A $\mathbb{Q}$-divisor $C$ on $Z$ is said to be $\tau$-equivalent to zero relative to $T$ (or $\tau$-equivalent to zero if there is no ambiguity on the base scheme $T$ ) if there exists $n \in \mathbb{Z} \backslash\{0\}$ such that $n C$ is a divisor on $Z$ which is $\tau$-equivalent to zero relative to $T$ (see Section 2.1). The group of classes of $\mathbb{Q}$-divisors on $Z$ which are $\tau$-equivalent to zero relative to $T$, modulo the principal divisors, will be denoted by $\operatorname{Pic}^{\mathbb{Q}, \tau}(Z / T)$.

When $Z=\bar{A}$, the restriction to the generic fiber induces an injective morphism

$$
\operatorname{Pic}^{\mathbb{Q}, \tau}(\bar{A} / R) \hookrightarrow \operatorname{Pic}_{A_{K} / K}^{\tau}(K)=\operatorname{Pic}_{A_{K} / K}^{0}(K)=A_{K}^{\prime}(K) .
$$

The fact that $\operatorname{Pic}_{A_{K} / K}^{\tau}(K)=\operatorname{Pic}_{A_{K} / K}^{0}(K)$ can be found in [Mumford 1974, (v) p. 75]. To see that the above morphism is injective, let $(C)$ be in its kernel. After modifying $C$ by a principal divisor if necessary, we can assume that $C_{K}=0$, that is, the support of $C$ is contained in the special fiber $\bar{A}_{k}$ of $\bar{A} / R$. Let $n$ be a nonzero integer such that $n C$ is a divisor on $\bar{A}$ which is $\tau$-equivalent to zero relative to $R$. As $\bar{A}_{k}$ admits at least one irreducible component $\Gamma$ with multiplicity 1 (the component containing the unit element of $A_{k}$ ), the vertical divisor $n C$ is principal [Raynaud 1970, 6.4.1 $3]$. In other words, there exists an integer $m$ such that $n C=m \operatorname{div}(\pi)$, where $\pi$ is a uniformizing element of $R$. Taking the associated cycles, and comparing the coefficients of $\Gamma$, we obtain that $n$ divides $m$. Consequently, the $\mathbb{Q}$-divisor $C$ is a principal divisor, whence the injectivity. 
By definition, the group $\operatorname{Pic}^{\mathbb{Q}, \tau}(\bar{A} / R)$ contains the group $\operatorname{Pic}^{0}(\bar{A} / R)$ of classes of divisors on $\bar{A}$ which are algebraically equivalent to zero relative to $R$, modulo principal divisors. Now, when $R$ is complete with algebraically closed residue field, we know from [Pepin 2011, Corollary 3.14] that the image of the composition

$$
\operatorname{Pic}^{0}(\bar{A} / R) \hookrightarrow \operatorname{Pic}^{\mathbb{Q}, \tau}(\bar{A} / R) \hookrightarrow A_{K}^{\prime}(K)
$$

contains the subgroup $\left(A^{\prime}\right)^{0}(R)$ of $A_{K}^{\prime}(K)$.

Conversely, we will show that Grothendieck's duality statement for $A$ and $A^{\prime}$ is equivalent to the following assertion: the image of $\mathrm{Pic}^{\mathbb{Q}, \tau}(\bar{A} / R) \hookrightarrow A_{K}^{\prime}(K)$ is contained in the subgroup $\left(A^{\prime}\right)^{0}(R)$.

Theorem 3.2.1. Let $R$ be a complete discrete valuation ring with algebraically closed residue field $k$ and fraction field $K$. Let $A_{K}$ be an abelian variety over $K$, with dual $A_{K}^{\prime}$. Let $A$ and $A^{\prime}$ be the Néron models of $A_{K}$ and $A_{K}^{\prime}$, respectively, over $R$. Let $\bar{A}$ be a proper flat normal model of $A_{K}$ over $R$, equipped with a dense open $R$-immersion $A \rightarrow \bar{A}$, such that the induced map $\operatorname{Pic}(\bar{A}) \rightarrow \operatorname{Pic}(A)$ is surjective. Let $\operatorname{Pic}^{\mathbb{Q}, \tau}(\bar{A} / R)$ be the group of $\mathbb{Q}$-divisors on $\bar{A}$ which are $\tau$-equivalent to zero relative to $R$, modulo the principal divisors. Then, the duality statement recalled in 3.1 is equivalent to the following:

The image of the restriction map $\operatorname{Pic}^{\mathbb{Q}, \tau}(\bar{A} / R) \hookrightarrow A_{K}^{\prime}(K)$ is contained in the subgroup $\left(A^{\prime}\right)^{0}(R)$.

Let $\operatorname{Pic}^{0}(\bar{A} / R)$ be the group of divisors on $\bar{A}$ which are algebraically equivalent to zero relative to $R$, modulo the principal divisors. Then, when the duality statement is true, the inclusion $\operatorname{Pic}^{0}(\bar{A} / R) \hookrightarrow \operatorname{Pic}^{\mathbb{Q}, \tau}(\bar{A} / R)$ is an equality, and there is a canonical commutative diagram

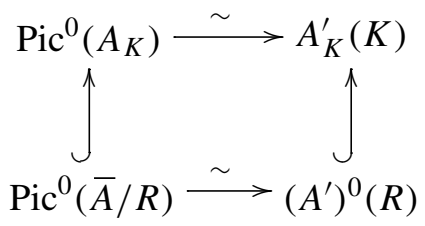

where the vertical maps are injective, and the horizontal maps are bijective.

See the end of Section 3.4 for the proof.

Remark 3.2.2. With the notation of Theorem 3.2.1, the canonical morphisms of abstract groups

$$
\operatorname{Pic}^{0}\left(A_{K}\right) \rightarrow \operatorname{Pic}_{A_{K} / K}^{0}(K), \quad \operatorname{Pic}^{0}(\bar{A} / R) \rightarrow \operatorname{Pic}_{\bar{A} / R}^{0}(R)
$$

are isomorphisms. For the second one, note that $\operatorname{Pic}_{\bar{A} / R}$ can be defined using the étale topology, and that $R$ is strictly henselian. Note also that, when $\bar{A}$ is locally factorial (e.g., regular), the group $\operatorname{Pic}^{\mathbb{Q}, \tau}(\bar{A} / R)$ coincides with the group $\operatorname{Pic}^{\tau}(\bar{A} / R)$ 
of divisors on $\bar{A}$ which are $\tau$-equivalent to zero relative to $R$, modulo the principal divisors, which in turn can be identified with the group $\operatorname{Pic}_{\bar{A} / R}^{\tau}(R)$.

The last assertion of Theorem 3.2.1 provides a refinement of [Pepin 2011, Corollary 3.14] in the present case $X=\bar{A}$. Here, when Grothendieck's duality holds, we obtain a necessary and sufficient condition for an invertible sheaf which is algebraically equivalent to zero on $A_{K}$ to extend into an invertible sheaf on $\bar{A}$ which is algebraically equivalent to zero relative to $R$ : the corresponding point $a_{K}^{\prime} \in A_{K}^{\prime}(K)$ must extend in the identity component of $A^{\prime}$. Thus, conjecturally, the group $\left(A^{\prime}\right)^{0}(R)$ parametrizes the invertible sheaves on $\bar{A}$ which are algebraically equivalent to zero relative to $R$.

To make the link between Grothendieck's duality for $A$ and $A^{\prime}$, and algebraic equivalence on $\bar{A}$, we need some preparation about nonrational 0 -cycles on $A_{K}$, especially those which are supported on inseparable points over $K$.

3.3. About nonrational 0-cycles on abelian varieties. Let $K$ be a field, and denote by $\bar{K}$ its algebraic closure. Let $A_{K}$ be an abelian variety over $K$. Let $d$ be a positive integer and let $\mathrm{Hilb}_{A_{K} / K}^{d}$ be the Hilbert scheme of points of degree $d$ on $A_{K}$. The Grothendieck-Deligne norm map

$$
\sigma_{d}: \operatorname{Hilb}_{A_{K} / K}^{d} \rightarrow A_{K}^{(d)}
$$

defined in [SGA 4 III 1973, (6.3.4.1) on p. $435=$ XVII-184] (see also [Bosch et al. 1990, pages 252-254]) maps $\mathrm{Hilb}_{A_{K} / K}^{d}$ to the $d$-fold symmetric product $A_{K}^{(d)}$. On the other hand, the map

$$
A_{K}^{d} \rightarrow A_{K}, \quad\left(x_{1}, \ldots, x_{d}\right) \mapsto x_{1}+\cdots+x_{d},
$$

induces a map

$$
m_{d}: A_{K}^{(d)} \rightarrow A_{K}
$$

Let us set

$$
\mathscr{Y}_{d}:=m_{d} \circ \sigma_{d}: \operatorname{Hilb}_{A_{K} / K}^{d} \rightarrow A_{K} .
$$

Let $a_{K} \in A_{K}$ be a closed point of degree $d$, that is to say, the residue field extension $K\left(a_{K}\right) / K$ has degree $d$. It corresponds to a rational point

$$
h\left(a_{K}\right) \in \operatorname{Hilb}_{A_{K} / K}^{d}(K) .
$$

We will need an explicit description of its image $\mathscr{S}_{d}\left(h\left(a_{K}\right)\right) \in A_{K}(K)$, when considered as an element of $A_{\bar{K}}(\bar{K})$.

Let us consider the artinian $\bar{K}$-scheme $a_{K} \otimes_{K} \bar{K}$. It is supported on some $a_{j} \in A_{\bar{K}}(\bar{K}), j=1, \ldots, s$, where $s$ is the separable degree of $K\left(a_{K}\right) / K$. The length 
of each local component of $a_{K} \otimes_{K} \bar{K}$ is equal to the inseparable degree of $K\left(a_{K}\right) / K$, and will be denoted by $l$. So the effective 0 -cycle associated to $a_{K} \otimes_{K} \bar{K}$ is

$$
\sum_{j=1}^{s} l\left[a_{j}\right] \in Z_{0}\left(A_{\bar{K}}\right) .
$$

We are going to show that

$$
\mathscr{S}_{d}\left(h\left(a_{K}\right)\right)=\sum_{j=1}^{s} l a_{j} \in A_{\bar{K}}(\bar{K}) .
$$

Note that, in particular, this will show that the right-hand-side of the equality belongs to $A_{K}(K)$.

Lemma 3.3.1. Let $C$ be an artinian algebra over an algebraically closed field $\bar{K}$. Let $C_{1}, \ldots, C_{s}$ be the local components of $C$, with respective lengths $l_{1}, \ldots, l_{s}$, and let $u_{j}: C_{j} \rightarrow \bar{K}$ be the canonical surjection from $C_{j}$ to its residue field. Then, for all

$$
c=\left(c_{1}, \ldots, c_{s}\right) \in C=C_{1} \times \cdots \times C_{s},
$$

the following formula holds for the norm of $c$ over $\bar{K}$ :

$$
N_{C / \bar{K}}(c)=\prod_{i=1}^{s}\left(u_{j}\left(c_{j}\right)\right)^{l_{j}} .
$$

Proof. We can assume that $C$ is local, with length $l$. Let $\mathfrak{m}$ be the maximal ideal of $C$. Let $n$ be the smallest integer such that $\mathfrak{m}^{n}=0$. Choose a basis $\mathscr{E}=\mathscr{E}_{0} \bigsqcup \cdots \bigsqcup^{\mathscr{E}_{n-1}}$ of $C$ over $\bar{K}$ which is adapted to the filtration

$$
0=\mathfrak{m}^{n} \subset \mathfrak{m}^{n-1} \subset \cdots \subset \mathfrak{m} \subset C,
$$

i.e., $\mathscr{E}_{i}$ is contained in $\mathfrak{m}^{i} \backslash \mathfrak{m}^{i+1}$ and induces a basis of the $\bar{K}$-vector space $\mathfrak{m}^{i} / \mathfrak{m}^{i+1}$.

Fix $c \in C$ and let $M$ be the matrix of multiplication-by-c in the basis $\mathscr{E}$. Write $c=\lambda+\epsilon$ with $\lambda \in \bar{K}$ and $\epsilon \in \mathfrak{m}$. Then $M$ is a $l \times l$ lower triangular matrix, with all diagonal entries equal to $\lambda$. Hence $N_{C / \bar{K}}(c)=\lambda^{l}$, as required.

We use the lemma to compute $\sigma_{d}\left(h\left(a_{K}\right)\right)$, considered as an element of $A_{\bar{K}}^{(d)}(\bar{K})$. Let $C$ be the $\bar{K}$-algebra of global sections of the scheme $a_{K} \otimes_{K} \bar{K}$. Set

$$
\operatorname{TS}_{\bar{K}}^{d}(C):=\left(C^{\otimes d}\right)^{\mathfrak{S}_{d}} \subseteq C^{\otimes d}
$$

where $\mathfrak{S}_{d}$ is the symmetric group acting on $C^{\otimes d}$ by permuting factors. By definition, the point $\sigma_{d}\left(h\left(a_{K}\right)\right) \in\left(a_{K} \otimes_{K} \bar{K}\right)^{(d)}(\bar{K}) \subset A_{\bar{K}}^{(d)}(\bar{K})$ corresponds to the unique $\bar{K}$ algebra homomorphism

$$
\mathrm{TS}_{\bar{K}}^{d}(C) \rightarrow \bar{K}, \quad c^{\otimes d} \mapsto N_{C / \bar{K}}(c) .
$$


Now, from Lemma 3.3.1, this homomorphism is induced by the point

$$
\left(a_{1}, \ldots, a_{1}, a_{2}, \ldots, a_{2}, \ldots, a_{s}, \ldots, a_{s}\right) \in A_{\bar{K}}^{d}(\bar{K}),
$$

where $a_{j}$ is repeated $l$ times.

Next, the element $\mathscr{Y}_{d}\left(h\left(a_{K}\right)\right) \in A_{\bar{K}}(\bar{K})$ is just the sum

$$
m_{d}\left(\sigma_{d}\left(h\left(a_{K}\right)\right)\right)=\sum_{j=1}^{s} l a_{j} \in A_{\bar{K}}(\bar{K}),
$$

as claimed.

Notation 3.3.2. The above $K$-morphisms $\mathscr{S}_{d}$ induce a homomorphism

$$
\mathscr{S}: Z_{0}\left(A_{K}\right) \rightarrow A_{K}(K)
$$

from the group of 0-cycles on $A_{K}$ to that of $K$-rational points: if $a_{K} \in A_{K}$ is a closed point of degree $d$, defining $h\left(a_{K}\right) \in \operatorname{Hilb}_{A_{K} / K}^{d}(K)$, then $\mathscr{Y}\left(\left[a_{K}\right]\right):=\mathscr{Y}_{d}\left(h\left(a_{K}\right)\right)$.

We will also need to "translate divisors on $A_{K}$ by nonrational points".

Let $\operatorname{Div}_{A_{K} / K}$ be the scheme of relative effective divisors on $A_{K}$ [FGA VI 1966, 4.1]. Fix a positive integer $d$ and consider the map

$$
A_{K}^{d} \times_{K} \operatorname{Div}_{A_{K} / K} \rightarrow \operatorname{Div}_{A_{K} / K}
$$

which is given by the functorial formula

$$
\left(\left(a_{1}, \ldots, a_{d}\right), D\right) \mapsto D_{a_{1}}+\cdots+D_{a_{d}},
$$

where $D_{a}$ is obtained from $D$ by translation by the section $a$. By symmetry, it induces a map

$$
A_{K}^{(d)} \times_{K} \operatorname{Div}_{A_{K} / K} \rightarrow \operatorname{Div}_{A_{K} / K}
$$

By composing with the norm map $\sigma_{d}$, the latter gives rise to a map

$$
\operatorname{Hilb}_{A_{K} / K}^{d} \times{ }_{K} \operatorname{Div}_{A_{K} / K} \rightarrow \operatorname{Div}_{A_{K} / K} .
$$

Let $a_{K} \in A_{K}$ be a closed point of degree $d$ and let $D_{K}$ be an effective divisor on $A_{K}$. Denote by $\left(D_{K}\right)_{a_{K}} \in \operatorname{Div}_{A_{K} / K}(K)$ the image of $\left(h\left(a_{K}\right), D_{K}\right)$ by the previous arrow. As above, write

$$
\sum_{r=1}^{d}\left[a_{\bar{K}, r}\right]
$$

for the 0 -cycle associated to $a_{K} \otimes_{K} \bar{K}$. In this expression, repetitions are allowed. Then, using the above computation of $\sigma_{d}\left(h\left(a_{K}\right)\right)$, we see that $\left(D_{K}\right)_{a_{K}}$, as an element 
of the group $\operatorname{Div}_{A_{\bar{K}} / \bar{K}}(\bar{K})$, is equal to

$$
\sum_{r=1}^{d}\left(D_{\bar{K}}\right)_{a_{\bar{K}, r}},
$$

where $D_{\bar{K}}$ denotes the pull-back of $D_{K}$ on $A_{\bar{K}}$. When $a_{K}$ is étale over $K$, it is easy to see that the latter divisor descends on $A_{K}$. But this turns out to be true in general because of the above construction. Moreover, this description shows that the formation of $\left(D_{K}\right)_{a_{K}}$ is additive in $D_{K}$. We can thus associate a divisor $\left(D_{K}\right)_{a_{K}}$ on $A_{K}$ to any divisor $D_{K}$ in the following way: identifying divisors on $A_{K}$ with 1-codimensional cycles, first use the above to define $\left(D_{K}\right)_{a_{K}}$ when $D_{K}$ is a prime cycle, and then extend by $\mathbb{Z}$-linearity.

Notation 3.3.3. If $c_{K}$ is a 0 -cycle on $A_{K}$ and $D_{K}$ a divisor on $A_{K}$, define the divisor $\left(D_{K}\right)_{c_{K}}$ on $A_{K}$ by $\mathbb{Z}$-linearity from the above situation where $c_{K}$ is a closed point.

3.4. Relative algebraic equivalence on semifactorial compactifications. Our goal in this subsection is to prove Theorem 3.2.1. So, until the end of the subsection, we fix a complete discrete valuation ring $R$ with algebraically closed residue field $k$ and fraction field $K$.

The starting point is the link between Grothendieck's pairing and Néron's pairing, which has been established by Bosch and Lorenzini: Grothendieck's pairing is the specialization of Néron's pairing.

Theorem 3.4.1 [Bosch and Lorenzini 2002, 4.4]. Keep the notation of Theorem 3.2.1. Moreover, let $\Phi_{A}$ and $\Phi_{A^{\prime}}$ be the groups of connected components of $A_{k}$ and $A_{k}^{\prime}$, respectively. On the one hand, consider Grothendieck's pairing [SGA 7 I 1972, IX 1.3]

$$
\langle\cdot, \cdot\rangle: \Phi_{A} \times \Phi_{A^{\prime}} \rightarrow \mathbb{Q} / \mathbb{Z}
$$

and on the other hand, consider Néron's pairing [Néron 1965, II 9.3]

$$
\langle\cdot, \cdot\rangle: Z_{0}^{0}\left(A_{K}\right) \times \operatorname{Div}^{0}\left(A_{K}\right) \rightarrow \mathbb{Q}
$$

(defined for $\left(c_{K}, D_{K}\right)$ when the supports of $c_{K}$ and $D_{K}$ are disjoint).

Let $\left(a, a^{\prime}\right) \in \Phi_{A} \times \Phi_{A^{\prime}}$. Fix a point $a_{K} \in A_{K}(K)$ specializing to a, and a divisor $D_{K}^{\prime} \in \operatorname{Div}^{0}\left(A_{K}\right)$ whose image in $A_{K}^{\prime}(K)$ specializes to $a^{\prime}$. Assume that $a_{K}$ and $0_{K}$ do not belong to the support of $D_{K}^{\prime}$. Then

$$
\left\langle a, a^{\prime}\right\rangle=-\left\langle\left[a_{K}\right]-\left[0_{K}\right], D_{K}^{\prime}\right\rangle \quad \bmod \mathbb{Z} .
$$

The following is a key result about the pairing $[\cdot, \cdot]$ defined in Proposition 2.1.3. 
Proposition 3.4.2. Let $X_{K}$ be a proper geometrically normal and geometrically connected scheme over $K$. Let $X$ be a proper flat normal semifactorial model of $X_{K}$ over $R$. Let $v$ be the number of irreducible components of the special fiber $X_{k}$. There exist some 0 -cycles of degree zero $c_{K, 1}, \ldots, c_{K, v}$ on $X_{K}$, with the following property:

If $D_{K}$ is a divisor on $X_{K}$ which is $\tau$-equivalent to zero, whose support is disjoint from those of the $c_{K, i}$, and if $\left[c_{K, i}, D_{K}\right]$ is an integer for all $i=1, \ldots, v$, then there exists $a \mathbb{Q}$-divisor on $X$ which is $\tau$-equivalent to zero relative $R$, with generic fiber $D_{K}$.

Proof. Let $U$ be the open subset of $X$ consisting of the regular points. As $X$ is normal, for any irreducible closed subset $C$ of codimension 1 in $X$, the intersection $C \cap U$ is a dense open subset of $C$. Furthermore, for any 1-codimensional cycle $C$ on $X$, the restriction $\left.C\right|_{U}$ is a divisor on $U$.

Next, let $\Gamma_{1}, \ldots, \Gamma_{v}$ be the reduced irreducible components of $X_{k}$. Let $\xi_{1}, \ldots, \xi_{v}$ be the generic points of $\Gamma_{1}, \ldots, \Gamma_{\nu}$. Set $d_{i}:=\operatorname{length}\left(O_{X_{k}, \xi_{i}}\right)$. From [Raynaud 1970, 7.1.2], there exists, for all $i=1, \ldots, v$, an $R$-immersion $u_{i}: Z_{i} \rightarrow U$, with $Z_{i}$ finite and flat over $R$, with rank $d_{i}$, such that $u_{i, k}\left(Z_{i, k}\right)$ is a point $x_{i, k}$ of $\Gamma_{i}$. Then the intersection multiplicity of $Z_{i}$ and $\Gamma_{j} \cap U$ is equal to 1 if $i=j$, and 0 otherwise. In particular, the generic fiber of $Z_{i}$ is a closed point $x_{K, i} \in U_{K}$ of degree $d_{i}$. Moreover, as $Z_{i}$ is proper over $R$, the immersion $Z_{i} \rightarrow X$ is closed. Finally, setting $d:=\operatorname{gcd}\left(d_{i}, i=1, \ldots, v\right)$, an appropriate $\mathbb{Z}$-linear combination of the $x_{K, i}$ provides a 0 -cycle $c_{K}$ on $X_{K}$ of degree $d$. We set

$$
c_{K, i}:=\left[x_{K, i}\right]-\frac{d_{i}}{d} c_{K} \in Z_{0}^{0}\left(X_{K}\right) .
$$

Let $D_{K} \in \operatorname{Div}^{\tau}\left(X_{K}\right)$ whose support is disjoint from those of the $c_{K, i}$. Choose $\Delta \in \operatorname{Div}^{\tau}(X / R)$ with a nonzero integer $n$ such that $\Delta_{K}=n D_{K}$. Denoting by $\bar{D}_{K}$ the schematic closure of $D_{K}$ in $X$, we can view $\Delta$ as a 1-codimensional cycle on $X$, and write

$$
\Delta=n \bar{D}_{K}+\sum_{i=1}^{v} n_{i} \Gamma_{i}
$$

for some integers $n_{1}, \ldots, n_{\nu}$. Set $V:=\sum_{i=1}^{v} n_{i} \Gamma_{i}$. As the schematic closures $\bar{c}_{K, i}$ of the $c_{K, i}$ in $X$ are contained in $U$ (by construction), the following computation is valid:

$$
\bar{c}_{K, i} \cdot \Delta=n\left(\bar{c}_{K, i} \cdot \bar{D}_{K}\right)+\left(\bar{x}_{K, i} \cdot V\right)-\frac{d_{i}}{d}\left(\bar{c}_{K} \cdot V\right)=n\left(\bar{c}_{K, i} \cdot \bar{D}_{K}\right)+n_{i}-\frac{d_{i}}{d}\left(\bar{c}_{K} \cdot V\right) .
$$


Assume that $\left[c_{K, i}, D_{K}\right]$ belongs to $\mathbb{Z}$. Then, the left-hand side of the above equality belongs to $n \mathbb{Z}$. Consequently, there exists $r_{i} \in \mathbb{Z}$ such that

$$
n r_{i}=n_{i}-\frac{d_{i}}{d}\left(\bar{c}_{K} \cdot V\right)
$$

Now, consider the vertical cycle (with integral coefficients)

$$
W:=\left(\bar{c}_{K} \cdot V\right) \frac{1}{d}\left[X_{k}\right]
$$

By definition,

$$
V-W=n \sum_{i=1}^{v} r_{i} \Gamma_{i}, \quad \text { that is, } \quad \Delta-W=n\left(\bar{D}_{K}-\sum_{i=1}^{\nu} r_{i} \Gamma_{i}\right) .
$$

The cycle $D:=\bar{D}_{K}-\sum_{i=1}^{v} r_{i} \Gamma_{i}$ is equal to $D_{K}$ on the generic fiber. This is a $\mathbb{Q}$-divisor on $X$ which is $\tau$-equivalent to zero because $d n D$ is a divisor on $X$ which is $\tau$-equivalent to zero.

Keep the notation of Proposition 3.4.2. Even if $X / R$ admits a section, so that $d$ is equal to 1 , the closed point $x_{K, i}$ is not rational as soon as the special fiber $X_{k}$ is not reduced at the generic point of the irreducible component $\Gamma_{i}$. Therefore, if we want to combine Theorem 3.4.1 and Proposition 3.4.2 when $X=\bar{A}$ (notation of Theorem 3.2.1), we need to compare the values of Néron's pairing on the abelian variety $A_{K}$ for 0 -cycles which are supported on nonrational points, with its values for 0 -cycles of the form $\left[a_{K}\right]-\left[0_{K}\right]$, with $a_{K} \in A_{K}(K)$. Here we will use the constructions of Section 3.3, together with some biduality argument. To take care of the conditions on supports involved in the computations of Néron's pairings, let us first note the following lemma.

Lemma 3.4.3. Let $A_{K}$ be an abelian variety over $K$ with dual $A_{K}^{\prime}$. Let $a_{K}^{\prime} \in A_{K}^{\prime}(K)$ and let $\mathscr{E}$ be a finite set of closed points of $A_{K}$. Then there exists a Poincaré divisor on $A_{K} \times_{K} A_{K}^{\prime}$, that is, a divisor such that the invertible sheaf $\mathbb{O}_{A_{K} \times{ }_{K} A_{K}^{\prime}}(P)$ is a Poincaré sheaf which is birigidified along $0_{K} \in A_{K}(K)$ and $0_{K}^{\prime} \in A_{K}^{\prime}(K)$, satisfying the following conditions:

(1) $P_{0_{K}}:=\left.P\right|_{0_{K} \times_{K} A_{K}^{\prime}}$ and $P_{0_{K}^{\prime}}:=\left.P\right|_{A_{K} \times_{K} 0_{K}^{\prime}}$ are well-defined and equal to zero.

(2) $P_{a_{K}^{\prime}}:=\left.P\right|_{A_{K} \times{ }_{K} a_{K}^{\prime}}$ is well-defined, and its support does not meet $\mathscr{E}$.

(3) For all $a_{K} \in \mathscr{E}, P_{a_{K}}:=\left.P\right|_{a_{K} \times_{K} A_{K}^{\prime}}$ is well-defined, and its support does not meet $\left\{0_{K}^{\prime}, a_{K}^{\prime}\right\}$.

Proof. Consider the finite set $\mathscr{F}$ whose elements are the following closed points of the product $A_{K} \times{ }_{K} A_{K}^{\prime}$ :

$$
a_{K} \times{ }_{K} 0_{K}^{\prime} \text { or } a_{K} \times{ }_{K} a_{K}^{\prime} \text {, with } a_{K} \in\left(\left\{0_{K}\right\} \coprod^{\mathscr{E}}\right) \text {. }
$$


Let $\mathscr{P}$ be a Poincaré sheaf on $A_{K} \times{ }_{K} A_{K}^{\prime}$, birigidified along $0_{K} \in A_{K}(K)$ and $0_{K}^{\prime} \in A_{K}^{\prime}(K)$. Choose an arbitrary divisor $Q$ such that $O_{A_{K} \times_{K} A_{K}^{\prime}}(Q) \simeq \mathscr{P}$. Using a moving lemma on the product $A_{K} \times{ }_{K} A_{K}^{\prime}$ if necessary [Liu 2002, 9.1.11], one can assume that the support of $Q$ is disjoint from the finite set $\mathscr{F}_{\text {. }}$ As $0_{K} \times{ }_{K} 0_{K}^{\prime} \in \mathscr{F}$, the divisors $\left.Q\right|_{0_{K} \times K} A_{K}^{\prime}$ and $\left.Q\right|_{A_{K} \times{ }_{K} 0_{K}^{\prime}}$ are well-defined, and are principal. Then

$$
P:=Q-p_{2}^{*}\left(\left.Q\right|_{0_{K} \times_{K} A_{K}^{\prime}}\right)-p_{1}^{*}\left(\left.Q\right|_{A_{K} \times_{K} 0_{K}^{\prime}}\right)
$$

(where $p_{1}: A_{K} \times_{K} A_{K}^{\prime} \rightarrow A_{K}$ and $p_{2}: A_{K} \times_{K} A_{K}^{\prime} \rightarrow A_{K}^{\prime}$ are the projections) is a Poincaré divisor again.

Now, let $a_{K} \in\left(\left\{0_{K}\right\} \coprod^{\mathscr{E}}\right)$. Then $a_{K} \times_{K} a_{K}^{\prime}$ does not belong to the support $\operatorname{Supp}(Q)$ of $Q$ because $a_{K} \times{ }_{K} a_{K}^{\prime} \in \mathscr{F}$. Next, $a_{K} \times_{K} a_{K}^{\prime} \notin \operatorname{Supp}\left(p_{2}^{*}\left(\left.Q\right|_{0_{K} \times_{K} A_{K}^{\prime}}\right)\right)$ : indeed, $0_{K} \times{ }_{K} a_{K}^{\prime} \in \mathscr{F}$ by definition, hence $0_{K} \times{ }_{K} a_{K}^{\prime} \notin \operatorname{Supp}(Q)$, and consequently $a_{K}^{\prime} \notin \operatorname{Supp}\left(\left.Q\right|_{0_{K} \times A_{K} A_{K}^{\prime}}\right)$. Finally $a_{K} \times_{K} a_{K}^{\prime} \notin \operatorname{Supp}\left(p_{1}^{*}\left(\left.Q\right|_{A_{K} \times_{K} 0_{K}^{\prime}}\right)\right)$, because otherwise $a_{K} \in \operatorname{Supp}\left(\left.Q\right|_{A_{K} \times{ }_{K} 0_{K}^{\prime}}\right)$ and $a_{K} \times{ }_{K} 0_{K}^{\prime} \in \operatorname{Supp}(Q)$, which is not the case because $a_{K} \times_{K} 0_{K}^{\prime} \in \mathscr{F}_{F}$. We have thus shown that the point $a_{K} \times_{K} a_{K}^{\prime}$ does not belong to the support of $P$. Similarly, the point $a_{K} \times{ }_{K} 0_{K}^{\prime}$ does not belong to the support of $P$. In conclusion:

(1) $\left.P\right|_{0_{K} \times{ }_{K} A_{K}^{\prime}}$ and $\left.P\right|_{A_{K} \times_{K} 0_{K}^{\prime}}$ are well-defined, and are equal to zero, by definition of $P$.

(2) $\left.P\right|_{A_{K} \times{ }_{K} a_{K}^{\prime}}$ is well-defined, and its support does not meet $\mathscr{E}$, because $a_{K} \times{ }_{K} a_{K}^{\prime} \notin$ $\operatorname{Supp}(P)$ for all $a_{K} \in \mathscr{E}$.

(3) $\left.P\right|_{a_{K} \times{ }_{K} A_{K}^{\prime}}$ is well-defined for all $a_{K} \in \mathscr{E}$, and its support does not meet $\left\{0_{K}^{\prime}, a_{K}^{\prime}\right\}$, because $a_{K} \times{ }_{K} a_{K}^{\prime} \notin \operatorname{Supp}(P)$ and $a_{K} \times{ }_{K} 0_{K}^{\prime} \notin \operatorname{Supp}(P)$ for all $a_{K} \in \mathscr{E}$.

We can now proceed to the announced comparison of some values of Néron's pairing.

Proposition 3.4.4. Let $A_{K}$ be an abelian variety with dual $A_{K}^{\prime}$. Let $c_{K} \in Z_{0}^{0}\left(A_{K}\right)$ and $D_{K}^{\prime} \in \operatorname{Div}^{0}\left(A_{K}\right)$. Assume that the support of $D_{K}^{\prime}$ is disjoint from that of $c_{K}$ and that of $\left[\mathscr{S}\left(c_{K}\right)\right]-\left[0_{K}\right]$ (Notation 3.3.2). Then the following relation between values of Néron's pairing on $A_{K}$ is true:

$$
\left\langle c_{K}, D_{K}^{\prime}\right\rangle \equiv\left\langle\left[\mathscr{S}\left(c_{K}\right)\right]-\left[0_{K}\right], D_{K}^{\prime}\right\rangle \quad \bmod \mathbb{Z} .
$$

Proof. Let $a_{K}^{\prime} \in A_{K}^{\prime}(K)$ corresponding to $D_{K}^{\prime}$. Let $\mathscr{E}$ be a finite set of closed points of $A_{K}$, containing the supports of $c_{K}$ and $\left[\mathscr{Y}\left(c_{K}\right)\right]-\left[0_{K}\right]$. From Lemma 3.4.3, there exists a Poincaré divisor $P$ satisfying the following conditions:

(1) $P_{0_{K}}:=\left.P\right|_{0_{K} \times A_{K} A_{K}^{\prime}}$ and $P_{0_{K}^{\prime}}:=\left.P\right|_{A_{K} \times{ }_{K} 0_{K}^{\prime}}$ are well-defined and equal to zero.

(2) $P_{a_{K}^{\prime}}:=\left.P\right|_{A_{K} \times K} a_{K}^{\prime}$ is well-defined, and its support does not meet $\mathscr{E}$.

(3) $P_{a_{K}}:=\left.P\right|_{a_{K} \times{ }_{K} A_{K}^{\prime}}$ is well-defined for all $a_{K} \in \mathscr{E}$, and its support does not meet $\left\{0_{K}^{\prime}, a_{K}^{\prime}\right\}$. 
Then, the divisors $D_{K}^{\prime}$ and $P_{a_{K}^{\prime}}$ are linearly equivalent. Consequently, we can assume $D_{K}^{\prime}=P_{a_{K}^{\prime}}$ (Proposition 2.2.5).

Write $c_{K}=c_{K}^{+}-c_{K}^{-}$where $c_{K}^{+}$and $c_{K}^{-}$are positive 0 -cycles with disjoint supports. Let $L / K$ be a finite field extension such that

$$
c_{K}^{+} \otimes_{K} L=\sum_{r=1}^{d}\left[a_{r,+}\right] \quad \text { and } \quad c_{K}^{-} \otimes_{K} L=\sum_{r=1}^{d}\left[a_{r,-}\right]
$$

where $d:=\operatorname{deg} c_{K}^{+}=\operatorname{deg} c_{K}^{-}$and with $a_{r,+}, a_{r,-}$ in $A_{L}(L)$ (repetitions allowed). Computing Néron's pairings over $K$ and over $L$ with normalized valuations, we get

$$
\left\langle c_{K}, P_{a_{K}^{\prime}}\right\rangle_{A_{K}}=\frac{1}{e_{L}}\left\langle\sum_{r=1}^{d}\left[a_{r,+}\right]-\sum_{r=1}^{d}\left[a_{r,-}\right],\left(P_{L}\right)_{a_{L}^{\prime}}\right\rangle_{A_{L}},
$$

where $P_{L}$ is the pull-back of $P$ over $L$, the point $a_{L}^{\prime} \in A_{L}^{\prime}(L)$ is the image of $a_{K}^{\prime} \in A_{K}^{\prime}(K)$ by the inclusion $A_{K}^{\prime}(K) \subseteq A_{L}^{\prime}(L)$, and $e_{L}$ is the ramification index of $L / K$. As $\left(P_{L}\right)_{0_{L}^{\prime}}=0$, the reciprocity law for Néron's pairing [Lang 1983, 11.4.2] ${ }^{1}$ asserts that the right-hand side of the equality is equal to the (well-defined) quantity

$$
\frac{1}{e_{L}}\left\langle\left[a_{L}^{\prime}\right]-\left[0_{L}^{\prime}\right], \sum_{r=1}^{d}\left(P_{L}\right)_{a_{r,+}}-\sum_{r=1}^{d}\left(P_{L}\right)_{a_{r,-}}\right\rangle_{A_{L}^{\prime}} .
$$

Now, with Notation 3.3.3, the divisor $\sum_{r=1}^{d}\left(P_{L}\right)_{a_{r,+}}-\sum_{r=1}^{d}\left(P_{L}\right)_{a_{r,-}}$ is precisely the pull-back over $L$ of the divisor $P_{c_{K}}$ on $A_{K}^{\prime}$. Furthermore, as the Poincaré map

$$
A_{L}(L) \rightarrow \operatorname{Pic}_{A_{L}^{\prime} / L}^{0}(L)
$$

is a group homomorphism, the divisors $P_{c_{K}}$ and $P_{S\left(c_{K}\right)}$ are linearly equivalent on $A_{L}^{\prime}$, and thus on $A_{K}^{\prime}$ (because $\operatorname{Pic}_{A_{K}^{\prime} / K}^{0}(K)$ is contained in $\operatorname{Pic}_{A_{K}^{\prime} / K}^{0}(L)$ ). Let $f \in K\left(A_{K}^{\prime}\right)$ be such that $P_{c_{K}}-P_{S\left(c_{K}\right)}=\operatorname{div}(f)$. As the normalized valuation on $K$ takes values in $\mathbb{Z}$, the (well-defined) pairing

$$
\frac{1}{e_{L}}\left\langle\left[a_{L}^{\prime}\right]-\left[0_{L}^{\prime}\right],(\operatorname{div}(f))_{L}\right\rangle_{A_{L}^{\prime}}=\left\langle\left[a_{K}^{\prime}\right]-\left[0_{K}^{\prime}\right], \operatorname{div}(f)\right\rangle_{A_{K}^{\prime}}
$$

is an integer. Consequently,

$$
\left\langle c_{K}, P_{a_{K}^{\prime}}\right\rangle_{A_{K}} \equiv\left\langle\left[a_{K}^{\prime}\right]-\left[0_{K}^{\prime}\right], P_{\mathscr{S}\left(c_{K}\right)}\right\rangle_{A_{K}^{\prime}} \bmod \mathbb{Z} .
$$

As $P_{0_{K}}=0$ and $P_{0_{K}^{\prime}}=0$, we conclude by using once again the reciprocity law.

\footnotetext{
${ }^{1}$ Here we use the reciprocity law in the case where the divisorial correspondence is the Poincare divisor $P_{L}$. By using a definition of Néron's pairing relying on the Poincaré biextension (see [Zahrin 1972, §5; Mazur and Tate 1983, §2]), the reciprocity law for $P_{L}$ is a direct consequence of the biduality of abelian varieties.
} 
We can now interpret Grothendieck's obstruction (Section 3.1) in terms of relative algebraic equivalence.

Theorem 3.4.5. Keep the notation of Theorem 3.2.1. Moreover, let $\Phi_{A}$ and $\Phi_{A^{\prime}}$ be the group of connected components of $A_{k}$ and $A_{k}^{\prime}$, respectively.

Let $a^{\prime} \in \Phi_{A^{\prime}}$. Lift $a^{\prime}$ to a point $a_{K}^{\prime} \in A_{K}^{\prime}(K)$, representing the linear equivalence class of a divisor $D_{K}^{\prime}$ on $A_{K}$. Then the homomorphism

$$
\left\langle\cdot, a^{\prime}\right\rangle: \Phi_{A} \rightarrow \mathbb{Q} / \mathbb{Z}
$$

induced by Grothendieck's pairing is identically zero if and only if $D_{K}^{\prime}$ can be extended to a $\mathbb{Q}$-divisor on $\bar{A}$ which is $\tau$-equivalent to zero relative to $R$.

Proof. Suppose that the obstruction $\left\langle\cdot, a^{\prime}\right\rangle$ vanishes. Choose 0 -cycles of degree zero $c_{K, 1}, \ldots, c_{K, v}$ on $A_{K}$ satisfying the conclusion of Proposition 3.4.2 when applied to the model $\bar{A} / R$ of $A_{K}$. To prove that $D_{K}^{\prime}$ extends to a $\mathbb{Q}$-divisor on $\bar{A}$ which is $\tau$-equivalent to zero, we can replace $D_{K}^{\prime}$ by any divisor on $A_{K}$ which is linearly equivalent to $D_{K}^{\prime}$. In particular, using moving lemma [Liu 2002, 9.1.11], we can assume that the support of $D_{K}^{\prime}$ does not meet the finite set

$$
\left\{0_{K}, S\left(c_{K, 1}\right), \ldots, S\left(c_{K, \nu}\right)\right\} \coprod_{i=1}^{v} \operatorname{Supp}\left(c_{K, i}\right) .
$$

Then, as $\left\langle\cdot, a^{\prime}\right\rangle=0$, we get from Bosch-Lorenzini's Theorem 3.4.1 that

$$
\left\langle\left[\mathscr{S}\left(c_{K, i}\right)\right]-\left[0_{K}\right], D_{K}^{\prime}\right\rangle \in \mathbb{Z}
$$

for all $i=1, \ldots, v$. Proposition 3.4.4 and Theorem 2.2.1 then imply that

$$
\left[c_{K, i}, D_{K}^{\prime}\right] \in \mathbb{Z}
$$

for all $i=1, \ldots, \nu$. Due to the choice of the $c_{K, i}$, the divisor $D_{K}^{\prime}$ can then be extended to a $\mathbb{Q}$-divisor on $\bar{A}$ which is $\tau$-equivalent to zero.

Conversely, suppose that there is a $\mathbb{Q}$-divisor $D^{\prime}$ on $\bar{A}$ which is $\tau$-equivalent to zero, with generic fiber $D_{K}^{\prime}$. To prove that $\left\langle\cdot, a^{\prime}\right\rangle=0$, we can assume that $0_{K}$ does not belong to the support of $D_{K}^{\prime}$, by adding to $D^{\prime}$ the divisor of a rational function on $\bar{A}$ if needed. Let $n^{\prime}$ be a nonzero integer such that $\Delta^{\prime}:=n^{\prime} D^{\prime}$ is a divisor on $\bar{A}$ which is $\tau$-equivalent to zero. For each $a_{K} \in A_{K}(K)$ which is not in the support of $D_{K}^{\prime}$, we get:

$$
\left[\left[a_{K}\right]-\left[0_{K}\right], D_{K}^{\prime}\right]=\frac{1}{n^{\prime}}\left(\left[\overline{a_{K}}\right]-\left[\overline{0_{K}}\right] \cdot \Delta^{\prime}\right)=\left(\left[\overline{a_{K}}\right]-\left[\overline{0_{K}}\right] \cdot D^{\prime}\right) \in \mathbb{Z} .
$$

The first equality holds by definition of the pairing $[\cdot, \cdot]$, and the second one is true because $\left[\overline{a_{K}}\right]-\left[\overline{0_{K}}\right]$ is contained in the regular locus of $\bar{A}$. Now observe that an element $a \in \Phi_{A}$ can always be lifted to a point $a_{K} \in A_{K}(K)$ which is not in 
the support of $D_{K}^{\prime}$. Thus, it follows from Theorem 2.2.1 and Bosch-Lorenzini's Theorem 3.4.1 that the obstruction $\left\langle\cdot, a^{\prime}\right\rangle$ vanishes.

Proof of Theorem 3.2.1. By biduality of abelian varieties, Grothendieck's duality statement is equivalent to the following: the obstruction $\left\langle\cdot, a^{\prime}\right\rangle$ vanishes if and only if $a^{\prime}=0$.

Suppose that this assertion is true. Let $(C) \in \operatorname{Pic}^{\mathbb{Q}, \tau}(\bar{A} / R)$ and let $a_{K}^{\prime}$ be its canonical image in $A_{K}^{\prime}(K)$. By Theorem 3.4.5, the obstruction $\left\langle\cdot, a^{\prime}\right\rangle$ vanishes. Hence $a^{\prime}=0$, that is, $a_{K^{\prime}} \in\left(A^{\prime}\right)^{0}(R)$.

Conversely, suppose that the canonical image of $\operatorname{Pic}^{\mathbb{Q}, \tau}(\bar{A} / R)$ in $A_{K}^{\prime}(K)$ is contained in $\left(A^{\prime}\right)^{0}(R)$. Let $a^{\prime} \in \Phi_{A^{\prime}}$, and assume that the corresponding obstruction $\left\langle\cdot, a^{\prime}\right\rangle$ vanishes. Choose a lifting $a_{K}^{\prime} \in A_{K}^{\prime}(K)$ of $a^{\prime}$. Then, by Theorem 3.4.5, the point $a_{K}^{\prime}$ belongs to the image of $\operatorname{Pic}^{\mathbb{Q}, \tau}(\bar{A} / R)$. In particular, it belongs to $\left(A^{\prime}\right)^{0}(R)$, and $a^{\prime}=0$.

Thus, we have proved that Grothendieck's conjecture is equivalent to the fact that the image of $\operatorname{Pic}^{\mathbb{Q}, \tau}(\bar{A} / R)$ in $A_{K}^{\prime}(K)$ is contained in $\left(A^{\prime}\right)^{0}(R)$. Now suppose that the conjecture is true. Then, from [Pepin 2011, Corollary 3.14], we obtain isomorphisms

$$
\operatorname{Pic}^{0}(\bar{A} / R) \stackrel{\sim}{\longrightarrow} \operatorname{Pic}^{\mathbb{Q}, \tau}(\bar{A} / R) \stackrel{\sim}{\longrightarrow}\left(A^{\prime}\right)^{0}(R) .
$$

The last assertion of Theorem 3.2.1 follows.

\section{Grothendieck's pairing for Jacobians}

4.1. Statement of the results. Let $R$ be a complete discrete valuation ring with algebraically closed residue field $k$ and fraction field $K$. Let $X_{K}$ be a proper smooth geometrically connected curve over $K$, and let $J_{K}:=\mathrm{Pic}_{X_{K} / K}^{0}$ be its Jacobian. Denote by $J$ and $J^{\prime}$ the Néron models of $J_{K}$ and $J_{K}^{\prime}$ over $R$, respectively, and $\Phi_{J}$ and $\Phi_{J^{\prime}}$ the groups of connected components of the special fiber of $J / R$ and $J^{\prime} / R$, respectively. Theorems 3.4.1 and 2.2.1 describe Grothendieck's pairing associated to $J_{K}$ in terms of intersection multiplicities on some compactification $\bar{J}$ of $J$. It is natural to wonder if these computations can be replaced by intersection computations on a proper flat regular model $X$ of $X_{K}$.

Assume that $X_{K}(K)$ is nonempty. In this case, the curve $X_{K}$ can be embedded into $J_{K}$, and can be used to define a classical theta divisor on $J_{K}$. Then, using Theorem 3.4.1, Bosch and Lorenzini described Grothendieck's pairing associated to $J_{K}$ in terms of the Néron pairing on $X_{K}$, and so in terms of intersection multiplicities on $X$, thanks to Gross's and Hriljac's Theorems [Gross 1986; Hriljac 1985]. Their precise result is as follows. Let $M$ be the intersection matrix of the special fiber of $X / R$ : if $\Gamma_{1}, \ldots, \Gamma_{v}$ are the irreducible components of $X_{k}$ equipped with their reduced scheme structure, the $(i, j)$-th entry of $M$ is the intersection number $\left(\Gamma_{i} \cdot \Gamma_{j}\right)$. Denote by $\Phi_{M}$ the torsion part of the cokernel of $M: \mathbb{Z}^{v} \rightarrow \mathbb{Z}^{v}$. According to 
Raynaud's work on the sheaf $\operatorname{Pic}_{X / S}$, there is a canonical isomorphism $\Phi_{J}=\Phi_{M}$; see [Bosch et al. 1990, 9.6/1]. Now, on the product $\Phi_{M} \times \Phi_{M}$, there is the canonical pairing

$$
\begin{aligned}
\langle\cdot, \cdot\rangle_{M}: \Phi_{M} \times \Phi_{M} & \rightarrow \mathbb{Q} / \mathbb{Z} \\
\left(\bar{T}, \bar{T}^{\prime}\right) & \mapsto\left({ }^{t} S / n\right) M\left(S^{\prime} / n^{\prime}\right) \quad \bmod \mathbb{Z}
\end{aligned}
$$

for any $n, n^{\prime} \in \mathbb{Z} \backslash\{0\}$ and $S, S^{\prime} \in \mathbb{Z}^{v}$ such that $M S=n T, M S^{\prime}=n^{\prime} T^{\prime}$. Now let $\left(a, a^{\prime}\right) \in \Phi_{J} \times \Phi_{J^{\prime}}$. By identifying $J_{K}$ and $J_{K}^{\prime}$ with the help of the opposite of the canonical principal polarization defined by a theta divisor, Grothendieck's pairing of $a$ and $a^{\prime}$ can be computed by the formula

$$
\left\langle a, a^{\prime}\right\rangle=\left\langle a, a^{\prime}\right\rangle_{M}
$$

[Bosch and Lorenzini 2002, Theorem 4.6].

Now assume that $X_{K}(K)$ is empty. Choosing a field extension $L / K$ such that $X_{K}(L)$ is nonempty, one can consider a theta divisor on $J_{L}$, and it is a classical fact that the associated canonical principal polarization is defined over $K$. Using its opposite, one can still identify $\Phi_{J}$ with $\Phi_{J^{\prime}}$, and thus $\Phi_{J^{\prime}}$ with $\Phi_{M}$ (as $k$ is algebraically closed, the identification $\Phi_{J}=\Phi_{M}$ holds without assuming that $X_{K}(K)$ is nonempty). Then the authors of [Bosch and Lorenzini 2002] ask if both pairings $\langle\cdot, \cdot\rangle$ and $\langle\cdot, \cdot\rangle_{M}$ still coincide in this situation [loc. cit., Remark 4.9]. In [Lorenzini 2008, Theorem 3.4], Lorenzini gives a positive answer to this question when the special fiber of $X / R$ admits two irreducible components $C_{i}$ and $C_{j}$ with multiplicities $d_{i}$ and $d_{j}$ such that $\left(C_{i} \cdot C_{j}\right)>0$ and $\operatorname{gcd}\left(d_{i}, d_{j}\right)=1$. Here we show that this result still holds if we only assume that the global gcd of the multiplicities of the irreducible components of $X_{k}$ is equal to 1 . Note that, due to the hypotheses on $R$ and on $X$, this global gcd coincides with the index of the curve $X_{K}$, that is, the smallest positive degree of a divisor on $X_{K}$ [Raynaud 1970, 7.1.6 1].

Theorem 4.1.1. Let $R$ be a complete discrete valuation ring with algebraically closed residue field $k$ and fraction field $K$. Let $X_{K}$ be a proper smooth geometrically connected curve over $K$, with index $d$. Let $J_{K}$ be the Jacobian of $X_{K}$, identified with its dual using the opposite of its canonical principal polarization. Let $X / R$ be a proper flat regular model of $X_{K}$. The following relation between Grothendieck's pairing for $J_{K}$ and the above pairing defined by the intersection matrix $M$ of $X_{k}$ is true:

$$
d\left\langle a, a^{\prime}\right\rangle=d\left\langle a, a^{\prime}\right\rangle_{M} .
$$

In particular, we get the following partial answers to Grothendieck's conjecture [SGA 7 I 1972, IX 1.3] in this case:

Corollary 4.1.2. Keep the notation of Theorem 4.1.1. Then:

- The kernel of Grothendieck's pairing for $J_{K}$ is killed by $d$. 
- If $d$ is prime to the characteristic of $k$, then Grothendieck's pairing for $J_{K}$ is perfect.

Proof. From [Bosch and Lorenzini 2002, Theorem 1.3], the pairing $\langle\cdot, \cdot\rangle_{M}$ is a perfect duality. So the first point follows directly from Theorem 4.1.1. For the second point, denote by $p$ the characteristic of $k$. Then Grothendieck's pairing is perfect when restricted to the prime-to- $p$ parts of the component groups: [SGA 7 I 1972, IX 11.3; Bertapelle 2001, Theorem 1]. Consequently, the second point follows again from the perfectness of $\langle\cdot, \cdot\rangle_{M}$ and Theorem 4.1.1.

4.2. Proof of Theorem 4.1.1. Here are two lemmas to prepare the proof of the theorem.

Recall that, as $R$ is complete with algebraically closed residue field, a classical result of Lang asserts that the Brauer group of $K$ is zero, whence $\operatorname{Pic}^{0}\left(X_{K}\right)=J_{K}(K)$.

Lemma 4.2.1. Let $a, a^{\prime} \in \Phi_{J}=\Phi_{M}$, and choose divisors $D_{K}, D_{K}^{\prime}$ on $X_{K}$ with disjoint supports, such that $a_{K}:=\left(D_{K}\right), a_{K}^{\prime}:=\left(D_{K}^{\prime}\right) \in J_{K}(K)=\operatorname{Pic}^{0}\left(X_{K}\right)$ specialize to $a, a^{\prime}$. The relationship between the pairing $\langle\cdot, \cdot\rangle_{M}$ and Néron's pairing on $X_{K}$ is given by:

$$
\left\langle a, a^{\prime}\right\rangle_{M}=-\left\langle D_{K}, D_{K}^{\prime}\right\rangle \quad \bmod \mathbb{Z} .
$$

Proof. This is an immediate consequence of the definitions, and of the description of Néron's pairing for the curve $X_{K}$ in terms of intersection multiplicities on $X$. Indeed, let $\rho: \operatorname{Pic}(X) \rightarrow \mathbb{Z}^{v}$ be the degree morphism $(Z) \mapsto\left(Z \cdot \Gamma_{i}\right)_{i=1, \ldots, \nu}$. Denote by $\overline{D_{K}}$ the schematic closure of $D_{K}$ in $X$. By definition of Raynaud's isomorphism $\Phi_{J}=\Phi_{M}$, the image of $\rho\left(\bar{D}_{K}\right) \in \mathbb{Z}^{v}$ in $\mathbb{Z}^{v} / \operatorname{Im} M$ is contained in the torsion part $\Phi_{M}$, and the resulting element is precisely the image of $a \in \Phi_{J}$ under the isomorphism. In particular, there are $n, n^{\prime} \in \mathbb{Z} \backslash\{0\}$ and $S, S^{\prime} \in \mathbb{Z}^{v}$ such that $M S=n \rho\left(\bar{D}_{K}\right), M S^{\prime}=n^{\prime} \rho\left(\bar{D}_{K}^{\prime}\right)$, and by definition of the symmetric pairing $\langle\cdot, \cdot\rangle_{M}$, we get

$$
\left\langle a, a^{\prime}\right\rangle_{M}=\left({ }^{t} S^{\prime} / n^{\prime}\right) \rho\left(\bar{D}_{K}\right) \quad \bmod \mathbb{Z} .
$$

Under the identification $\bigoplus_{i=1}^{v} \mathbb{Z} \Gamma_{i} \simeq \mathbb{Z}^{v}$, the right-hand side can also be written as an intersection multiplicity:

$$
\left\langle a, a^{\prime}\right\rangle_{M}=\frac{1}{n^{\prime}}\left(\bar{D}_{K} \cdot S^{\prime}\right)=-\frac{1}{n^{\prime}}\left(\bar{D}_{K} \cdot\left(n^{\prime} \bar{D}_{K}^{\prime}-S^{\prime}\right)\right) \quad \in \mathbb{Q} / \mathbb{Z} .
$$

Now, the equality $M S^{\prime}=n^{\prime} \rho\left(\bar{D}_{K}^{\prime}\right)$ means that the divisor $n^{\prime} \bar{D}_{K}^{\prime}-S^{\prime}$ on $X$ is algebraically equivalent to zero relative to $R$ ([Bosch et al. 1990] 9.2/13). Applying Theorem 2.2.1 to the curve $X_{K}$, we conclude that

$$
\left\langle a, a^{\prime}\right\rangle_{M}=-\left[D_{K}, D_{K}^{\prime}\right]=-\left\langle D_{K}, D_{K}^{\prime}\right\rangle \in \mathbb{Q} / \mathbb{Z} .
$$


Next, the index $d$ of $X_{K}$ divides $g-1$ where $g$ is the genus of $X_{K}$ [Raynaud 1970, 9.5.1]. Let us fix a divisor $E$ of degree $d$ on $X_{K}$, and consider the linear equivalence class of divisors of degree $g-1$ given by

$$
t_{K}:=(g-1) d^{-1}(E) \in \operatorname{Pic}_{X_{K} / K}^{g-1}(K) .
$$

The canonical image of the $(g-1)$-fold symmetric product $X_{K}^{(g-1)}$ in $\mathrm{Pic}_{X_{K} / K}^{g-1}$ can be translated by $t_{K}$ to a divisor on $J_{K}$, which we will denote by $\Theta$. Then, by extending $K$ and reducing to the case where $X_{K}(K)$ is nonempty, one sees that the canonical principal polarization $\varphi$ of $J_{K}$ can be written explicitly here as $\varphi(z)=-\left(\Theta_{z}-\Theta\right)$, where $\Theta_{z}$ is obtained from $\Theta$ by translation by the point $z$. On the other hand, denoting by $\Delta$ the diagonal of $X_{K} \times{ }_{K} X_{K}$, the divisor $d \Delta-E \times{ }_{K} X_{K}$ on $X_{K} \times_{K} X_{K}$ defines an element of $\operatorname{Pic}_{X_{K} / K}^{0}\left(X_{K}\right)$, hence a $K$-morphism $h: X_{K} \rightarrow \operatorname{Pic}_{X_{K} / K}^{0}=J_{K}$.

Lemma 4.2.2. The following diagram of $K$-morphisms is commutative:

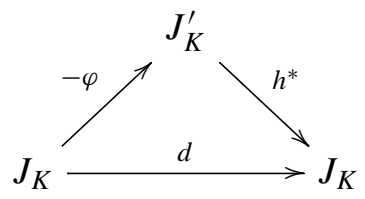

The commutativity can be stated as follows. Let $z \in J_{K}(\bar{K})$. Let $Z$ be any divisor of degree 0 on $X_{\bar{K}}$, whose linear equivalence class $(Z)$ corresponds to $z$ via the canonical isomorphism $\operatorname{Pic}^{0}\left(X_{\bar{K}}\right)=J_{K}(\bar{K})$. Then the following relation holds:

$$
h^{*}\left(\Theta_{z}-\Theta\right)=d(Z) \in \operatorname{Pic}^{0}\left(X_{\bar{K}}\right)=J_{K}(\bar{K}) .
$$

In particular, there is a nonempty open subset $U_{K}$ of $J_{K}$ such that $h^{*} \Theta_{z}$ is a welldefined divisor on $X_{K}$ for all $z \in U_{K}(K)$, and whose degree does not depend on the point $z$.

Proof. To check that the diagram is commutative, one can replace $K$ by its algebraic closure, and so we can assume that $K$ is algebraically closed. As the pull-back by the multiplication-by- $d$ on $J_{K}$ acts as multiplication-by- $d$ on the group $\operatorname{Pic}^{0}\left(J_{K}\right)$, the lemma then follows from the classical situation where $X_{K}$ can be embedded into $J_{K}$ using a rational point of $X_{K}$.

Proof of Theorem 4.1.1. Let $\left(a, a^{\prime}\right) \in \Phi_{J} \times \Phi_{J}$. Choose a point $a_{K} \in J_{K}(K)$ which specializes to $a \in \Phi_{J}$. The point $a_{K}$ corresponds, under the equality $J_{K}(K)=$ $\operatorname{Pic}^{0}\left(X_{K}\right)$, to the linear equivalence class of a divisor $D(a)_{K}$ of degree 0 on $X_{K}$. Write $D(a)_{K}=D(a)_{K}^{+}-D(a)_{K}^{-}$with $D(a)_{K}^{+}$and $D(a)_{K}^{-}$positive with disjoint supports. Let $L / K$ be a finite field extension such that

$$
D(a)_{K}^{+} \otimes_{K} L=\sum_{r=1}^{\alpha}\left[a_{r,+}\right] \quad \text { and } \quad D(a)_{K}^{-} \otimes_{K} L=\sum_{r=1}^{\alpha}\left[a_{r,-}\right],
$$


where $\alpha:=\operatorname{deg} D(a)_{K}^{+}=\operatorname{deg} D(a)_{K}^{-}$and with $a_{r,+}, a_{r,-}$ in $X_{L}(L)$ (repetitions allowed).

Next, still denoting by $U_{K}$ the open subset of $J_{K}$ provided by Lemma 4.2.2, one can find $a_{K}^{\prime}$ and $z_{K}$ in $U_{K}(K)$ specializing respectively to $a^{\prime}$ and 0 in $\Phi_{J}$, and such that

$$
\begin{aligned}
& d a_{K}, \quad 0_{K} \notin \operatorname{Supp}\left(\Theta_{a_{K}^{\prime}}-\Theta_{z_{K}}\right) \\
& \bar{a}_{r,+}, \bar{a}_{r,-} \notin \operatorname{Supp}\left(\left(\Theta_{a_{K}^{\prime}}-\Theta_{z_{K}}\right)_{L}\right) \quad \text { for all } r=1, \ldots, \alpha,
\end{aligned}
$$

where $\bar{a}_{r,+}:=h\left(a_{r,+}\right)$ and $\bar{a}_{r,-}:=h\left(a_{r,-}\right)$. The points $a_{K}^{\prime}$ and $z_{K}$ correspond to the classes of some divisors $D\left(a^{\prime}\right)_{K}$ and $D(0)_{K}$ on $X_{K}$, under the identification $J_{K}(K)=\operatorname{Pic}^{0}\left(X_{K}\right)$. From Lemma 4.2.2, we get:

$$
h^{*}\left(\Theta_{a_{K}^{\prime}}-\Theta_{z_{K}}\right)=d\left(D\left(a^{\prime}\right)_{K}-D(0)_{K}\right)=d\left(a_{K}^{\prime}-z_{K}\right)
$$

in $\operatorname{Pic}^{0}\left(X_{K}\right)=J_{K}(K)$. And by construction, the $K$-point $d\left(a_{K}^{\prime}-z_{K}\right)$ of $J_{K}$ specializes to $d a^{\prime} \in \Phi_{J}$. As a consequence, Lemma 4.2.1 provides the formula:

$$
\left\langle a, d a^{\prime}\right\rangle_{M}=-\left\langle D(a)_{K}, h^{*}\left(\Theta_{a_{K}^{\prime}}-\Theta_{z_{K}}\right)\right\rangle_{X_{K}} \bmod \mathbb{Z}
$$

(note that $h^{*}\left(\Theta_{a_{K}^{\prime}}-\Theta_{z_{K}}\right)$ is a well-defined divisor, and not only a class, because $\left.a_{K}^{\prime}, z_{K} \in U_{K}(K)\right)$.

Still working with normalized valuations to compute Néron's pairing, and using functoriality, we obtain:

$$
\left\langle a, d a^{\prime}\right\rangle_{M}=-\frac{1}{e_{L}}\left\langle\sum_{r=1}^{\alpha}\left[\bar{a}_{r,+}\right]-\left[\bar{a}_{r,-}\right],\left(\Theta_{a_{K}^{\prime}}-\Theta_{z_{K}}\right)_{L}\right\rangle_{J_{L}} \bmod \mathbb{Z},
$$

where $e_{L}$ is the ramification index of $L / K$. Then we apply the reciprocity law for Néron's pairing with the divisorial correspondence $\left(\delta^{*} \Theta-p_{1}^{*} \Theta-p_{2}^{*} \Theta\right)_{L}$, where $\delta$, $p_{1}$ and $p_{2}: J_{K} \times_{K} J_{K} \rightarrow J_{K}$ are the difference map and the two projections, to get:

$$
\left\langle a, d a^{\prime}\right\rangle_{M}=-\frac{1}{e_{L}}\left\langle\left[a_{L}^{\prime}\right]-\left[z_{L}\right], \sum_{r=1}^{\alpha}\left(\Theta_{L}\right)_{\bar{a}_{r,+}}^{-}-\left(\Theta_{L}\right)_{\bar{a}_{r,-}}^{-}\right\rangle_{J_{L}} \bmod \mathbb{Z} .
$$

Here $\left(\Theta_{L}\right)^{-}$stands for $[-1]^{*}\left(\Theta_{L}\right)$.

Now, with Notation 3.3.3, the divisor $\sum_{r=1}^{\alpha}\left(\Theta_{L}\right)_{\bar{a}_{r,+}}^{-}-\left(\Theta_{L}\right)_{\bar{a}_{r,-}}^{-}$is the pull-back on $J_{L}$ of the divisor $\left(\Theta^{-}\right)_{h_{*} D(a)}$ defined on $J_{K}$. On the other hand,

$$
\begin{aligned}
\sum_{r=1}^{\alpha} \bar{a}_{r,+}-\bar{a}_{r,-} & =\sum_{r=1}^{\alpha}\left(d\left[a_{r,+}\right]-E_{L}\right)-\left(d\left[a_{r,-}\right]-E_{L}\right) \\
& =d\left(D(a)_{L}\right) \in J_{K}(L) \\
& =d a_{K} \in J_{K}(K) .
\end{aligned}
$$


Therefore the theorem of the square on $J_{L}$ shows that the two divisors $\left(\Theta^{-}\right)_{h_{*} D(a)}$ and $\Theta_{d a_{K}}^{-}-\Theta^{-}$on $J_{K}$ are linearly equivalent over $L$, hence also over $K\left(J_{K}^{\prime}(K)\right.$ injects into $J_{L}^{\prime}(L)$ ). From this observation, and the fact that the normalized valuation on $K$ takes values in $\mathbb{Z}$, we deduce that

$$
\left\langle a, d a^{\prime}\right\rangle_{M}=-\left\langle\left[a_{K}^{\prime}\right]-\left[z_{K}\right], \Theta_{d a_{K}}^{-}-\Theta^{-}\right\rangle_{J_{K}} \bmod \mathbb{Z} .
$$

Applying once more the reciprocity law, we find

$$
\left\langle a, d a^{\prime}\right\rangle_{M}=-\left\langle\left[d a_{K}\right]-\left[0_{K}\right], \Theta_{a_{K}^{\prime}}-\Theta_{z_{K}}\right\rangle \bmod \mathbb{Z} .
$$

Finally, note that $\left(\Theta_{a_{K}^{\prime}}-\Theta_{z_{K}}\right)=-\varphi\left(a_{K}^{\prime}-z_{K}\right) \in J^{\prime}(K)$ and $a_{K}^{\prime}-z_{K}$ specializes to $a^{\prime} \in \Phi_{J}$. Consequently, if we use $-\varphi$ to identify $J_{K}$ with its dual, Theorem 3.4.1 tells us that

$$
-\left\langle\left[d a_{K}\right]-\left[0_{K}\right], \Theta_{a_{K}^{\prime}}-\Theta_{z_{K}}\right\rangle=\left\langle d a, a^{\prime}\right\rangle \quad \bmod \mathbb{Z} .
$$

Whence $\left\langle a, d a^{\prime}\right\rangle_{M}=\left\langle d a, a^{\prime}\right\rangle$, as claimed.

\section{Acknowledgments}

I would like to thank Michel Raynaud for many enlightening discussions about Néron's and Grothendieck's pairings. I am indebted to Qing Liu for his valuable teaching of intersection theory on relative schemes. I am very grateful to Pascal Autissier, Siegfried Bosch, Dino Lorenzini and Laurent Moret-Bailly for their careful reading and for many helpful comments. I thank the referee for his meticulous reading and for many useful remarks.

\section{References}

[Bégueri 1980] L. Bégueri, Dualité sur un corps local à corps résiduel algébriquement clos, Mém. Soc. Math. France (N.S.) 4, 1980. MR 82k:12019 Zbl 0502.14016

[Bertapelle 2001] A. Bertapelle, "On perfectness of Grothendieck's pairing for the $l$-parts of component groups”, J. Reine Angew. Math. 538 (2001), 223-236. MR 2002k:14072 Zbl 1028.14017

[Bosch and Lorenzini 2002] S. Bosch and D. Lorenzini, "Grothendieck's pairing on component groups of Jacobians”, Invent. Math. 148:2 (2002), 353-396. MR 2003b:14053 Zbl 1061.14042

[Bosch et al. 1990] S. Bosch, W. Lütkebohmert, and M. Raynaud, Néron models, Ergeb. Math. Grenzgeb. (3) 21, Springer, Berlin, 1990. MR 91i:14034 Zbl 0705.14001

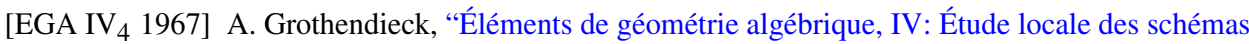
et des morphismes de schémas, IV", Inst. Hautes Études Sci. Publ. Math. 32 (1967), 5-361. MR 39 \#220 Zbl 0153.22301

[FGA VI 1966] A. Grothendieck, “Technique de descente et théorèmes d'existence en géométrie algébrique, VI: Les schémas de Picard, propriétés générales”, exposé 236 in Séminaire Bourbaki, 1961/1962, W. A. Benjamin, New York, 1966. Reprinted as pp. 221-243 in Séminaire Bourbaki 7, Soc. Math. France, Paris, 1995. MR 1611207 Zbl 0238.14015 
[Fulton 1998] W. Fulton, Intersection theory, 2nd ed., Ergeb. Math. Grenzgeb. (3) 2, Springer, Berlin, 1998. MR 99d:14003 Zbl 0885.14002

[Gross 1986] B. H. Gross, "Local heights on curves", pp. 327-339 in Arithmetic geometry (Storrs, CT, 1984), edited by G. Cornell and J. H. Silverman, Springer, New York, 1986. MR 861983 Zbl 0605.14027

[Hriljac 1985] P. Hriljac, "Heights and Arakelov's intersection theory", Amer. J. Math. 107:1 (1985), 23-38. MR 86c:14024 Zbl 0593.14004

[Lang 1983] S. Lang, Fundamentals of Diophantine geometry, Springer, New York, 1983. MR 85j: 11005 Zbl 0528.14013

[Lang 1988] S. Lang, Introduction to Arakelov theory, Springer, New York, 1988. MR 89m:11059 Zbl 0667.14001

[Liu 2002] Q. Liu, Algebraic geometry and arithmetic curves, Oxford Graduate Texts in Mathematics 6, Oxford University Press, 2002. MR 2003g:14001 Zbl 0996.14005

[Loerke 2009] K. Loerke, "Reduction of Abelian varieties and Grothendieck's pairing", preprint, 2009. arXiv 0909.4425v1

[Lorenzini 2008] D. Lorenzini, "Grothendieck's pairing for Jacobians and base change", J. Number Theory 128:6 (2008), 1448-1457. MR 2009b:14088 Zbl 1198.14042

[Mazur and Tate 1983] B. Mazur and J. Tate, "Canonical height pairings via biextensions", pp. 195-237 in Arithmetic and geometry, vol. 1, edited by M. Artin and J. Tate, Progress in Mathematics 35, Birkhäuser, Boston, MA, 1983. MR 85j:14081 Zbl 0574.14036

[Mumford 1974] D. Mumford, Abelian varieties, 2nd ed., Tata Inst. Fund. Res. Studies in Math. 5, Oxford University Press, London, 1974. Zbl 0326.14012

[Murre 1964] J. P. Murre, "On contravariant functors from the category of pre-schemes over a field into the category of Abelian groups (with an application to the Picard functor)", Inst. Hautes Études Sci. Publ. Math. 23 (1964), 5-43. MR 34 \#5836 Zbl 0142.18402

[Néron 1965] A. Néron, "Quasi-fonctions et hauteurs sur les variétés abéliennes", Ann. of Math. (2) 82 (1965), 249-331. MR 31 \#3424 Zbl 0163.15205

[Oort 1962] F. Oort, "Sur le schéma de Picard”, Bull. Soc. Math. France 90 (1962), 1-14. MR 25 \#2070 Zbl 0123.13901

[Pepin 2011] C. Pepin, "Modèles semi-factoriels et modèles de Néron", preprint, 2011, Available at https://perswww.kuleuven.be/ u0079577/semi-factoriel-final.pdf. To appear in Math. Ann.

[Raynaud 1970] M. Raynaud, "Spécialisation du foncteur de Picard”, Inst. Hautes Études Sci. Publ. Math. 38 (1970), 27-76. MR 44 \#227 Zbl 0207.51602

[SGA 4 III 1973] M. Artin, A. Grothendieck, and J. L. Verdier (editors), Théorie des topos et cohomologie étale des schémas. Tome 3. Exposes IX à XIX. (Séminaire de Géométrie Algébrique du Bois-Marie 1963-1964 = SGA 4), Lecture Notes in Math. 305, Springer, Berlin, 1973. MR 50 \#7132 Zbl 0245.00002

[SGA 6 1971] A. Grothendieck, P. Berthelot, and L. Illusie, Séminaire de Géométrie Algébrique du Bois Marie 1966/1967: Théorie des intersections et théorème de Riemann-Roch (SGA 6), Lecture Notes in Mathematics 225, Springer, Berlin, 1971. MR 50 \#7133 Zbl 218.14001

[SGA 7 I 1972] A. Grothendieck, Séminaire de Géométrie Algébrique du Bois Marie 1967/1969: Groupes de monodromie en géométrie algébrique, I (SGA 7), Lecture Notes in Mathematics 288 , Springer, Berlin, 1972. MR 50 \#7134 Zbl 0237.00013

[Werner 1997] A. Werner, “On Grothendieck's pairing of component groups in the semistable reduction case”, J. Reine Angew. Math. 486 (1997), 205-215. MR 98j:14058 Zbl 0872.14037 
[Zahrin 1972] J. G. Zahrin, "Néron pairing and quasicharacters", Math. USSR Izv. 6:3 (1972), 491503. Zbl 0254.14012

Communicated by Barry Mazur

Received 2011-02-19 Revised 2011-12-21 Accepted 2012-01-18

cedric.pepin@wis.kuleuven.be KU Leuven, Departement Wiskunde, Celestijnenlaan 200B, 3001 Heverlee, Belgium

https://perswww.kuleuven.be/ u0079577/ 


\section{Algebra \& Number Theory}

msp.berkeley.edu/ant

\section{EDITORS}

MANAGING EDITOR

Bjorn Poonen

Massachusetts Institute of Technology

Cambridge, USA

\author{
EDITORIAL BOARD CHAIR \\ David Eisenbud \\ University of California \\ Berkeley, USA
}

\section{BOARD OF EDITORS}

Georgia Benkart

Dave Benson

Richard E. Borcherds

John H. Coates

J-L. Colliot-Thélène

Brian D. Conrad

Hélène Esnault

Hubert Flenner

Edward Frenkel

Andrew Granville

Joseph Gubeladze

Ehud Hrushovski

Craig Huneke

Mikhail Kapranov

Yujiro Kawamata

János Kollár

Yuri Manin

Barry Mazur

Philippe Michel
University of Wisconsin, Madison, USA

University of Aberdeen, Scotland

University of California, Berkeley, USA

University of Cambridge, UK

CNRS, Université Paris-Sud, France

University of Michigan, USA

Freie Universität Berlin, Germany

Ruhr-Universität, Germany

University of California, Berkeley, USA

Université de Montréal, Canada

San Francisco State University, USA

Hebrew University, Israel

University of Virginia, USA

Yale University, USA

University of Tokyo, Japan

Princeton University, USA

Northwestern University, USA

Harvard University, USA

École Polytechnique Fédérale de Lausanne
Susan Montgomery

Shigefumi Mori

Raman Parimala

Jonathan Pila

Victor Reiner

Karl Rubin

Peter Sarnak

Joseph H. Silverman

Michael Singer

Vasudevan Srinivas

J. Toby Stafford

Bernd Sturmfels

Richard Taylor

Ravi Vakil

Michel van den Bergh

Marie-France Vignéras

Kei-Ichi Watanabe

Andrei Zelevinsky

Efim Zelmanov
University of Southern California, USA

RIMS, Kyoto University, Japan

Emory University, USA

University of Oxford, UK

University of Minnesota, USA

University of California, Irvine, USA

Princeton University, USA

Brown University, USA

North Carolina State University, USA

Tata Inst. of Fund. Research, India

University of Michigan, USA

University of California, Berkeley, USA

Harvard University, USA

Stanford University, USA

Hasselt University, Belgium

Université Paris VII, France

Nihon University, Japan

Northeastern University, USA

University of California, San Diego, USA

\section{PRODUCTION}

production@msp.org

Silvio Levy, Scientific Editor

See inside back cover or www.jant.org for submission instructions.

The subscription price for 2012 is US \$175/year for the electronic version, and \$275/year ( $\$ 40$ shipping outside the US) for print and electronic. Subscriptions, requests for back issues from the last three years and changes of subscribers address should be sent to Mathematical Sciences Publishers, Department of Mathematics, University of California, Berkeley, CA 94720-3840, USA.

Algebra \& Number Theory (ISSN 1937-0652) at Mathematical Sciences Publishers, Department of Mathematics, University of California, Berkeley, CA 94720-3840 is published continuously online. Periodical rate postage paid at Berkeley, CA 94704, and additional mailing offices.

ANT peer review and production are managed by EditFLOW ${ }^{\circledR}$ from Mathematical Sciences Publishers.

PUBLISHED BY

mathematical sciences publishers

http://msp.org/

A NON-PROFIT CORPORATION

Typeset in IATEX

Copyright ( 2012 by Mathematical Sciences Publishers 


\section{Algebra \& Number Theory}

\section{Volume $6 \quad$ No. $7 \quad 2012$}

On the rank of the fibers of rational elliptic surfaces

Cecília Salgado

Néron's pairing and relative algebraic equivalence

CÉDRIC PÉPIN

Free subalgebras of quotient rings of Ore extensions

JASON P. BELL and DANIEL ROGALSKI

Classes de cycles motiviques étales

1369

BRUNO KAHN

Higher-order Maass forms

1409

ROELOF BRUGGEMAN and NiKOLAOS DIAMANTIS

Log canonical thresholds, $F$-pure thresholds, and nonstandard extensions

Bhargav Bhatt, Daniel J. Hernández, Lance Edward Miller and Mircea MUSTAȚĂ

The semistable reduction problem for the space of morphisms on $\mathbb{P}^{n}$

ALON LEVY

Grothendieck's trace map for arithmetic surfaces via residues and higher adèles

MATTHEW MORROW

Crystalline extensions and the weight part of Serre's conjecture

Toby GeE, Tong LiU and DAVID SAVITT

Annihilating the cohomology of group schemes 